\title{
STABILITY OF THE TRAVELLING WAVE SOLUTION OF THE FITZHUGH-NAGUMO SYSTEM
}

BY

\author{
CHRISTOPHER K. R. T. JONES ${ }^{1}$
}

\begin{abstract}
Travelling wave solutions for the FitzHugh-Nagumo equations have been proved to exist, by various authors, close to a certain singular limit of the equations. In this paper it is proved that these waves are stable relative to the full system of partial differential equations; that is, initial values near (in the sup norm) to the travelling wave lead to solutions that decay to some translate of the wave in time. The technique used is the linearised stability criterion; the framework for its use in this context has been given by Evans [6-9]. The search for the spectrum leads to systems of linear ordinary differential equations. The proof uses dynamical systems arguments to analyse these close to the singular limit.
\end{abstract}

1. Introduction. Travelling waves play a central role in the theory of reaction-diffusion equations. Many techniques have been developed to find such waves, i.e., prove their existence; see Conley and Gardner [4], Gardner and Smoller [16], and Dunbar [5] for recent results. However, the equation of their stability relative to the PDE has remained fairly open. Scalar equations are now well understood; see Fife [12], Fife and McLeod [13], and Bramson [1]. For systems, the only fully established results involve assumptions on the nonlinearity that permit the application of a maximum principle type argument, i.e., some monotonicity; see Klaasen and Troy [19], and Gardner [15]. Feroe [11] has performed some numerical calculations on the stability problem for the FitzHugh-Nagumo equations with a special assumption of piecewise linearity on the nonlinear term.

In this paper I shall prove a stability result for the FitzHugh-Nagumo equations. These equations are a paradigm example of a system of equations to which the maximum principle is difficult to apply; see Terman [22].

The FitzHugh-Nagumo equations are the following system of reaction-diffusion equations:

$$
u_{t}=u_{x x}+f(u)-w, \quad w_{t}=\varepsilon(u-\gamma w) .
$$

The function

$$
f(u)=u(u-a)(1-u)
$$

is a cubic, where $a<1 / 2$. The constants $\varepsilon$ and $\gamma$ are positive. I shall be interested in the case $\varepsilon \ll 1$ and $\gamma \ll 1 ; \gamma$ is often assumed to be zero.

Received by the editors March 16, 1983 and, in revised form. January 30, 1984. 1980 Mathematics Subject Classification. Primary 35B35: Secondary 34B25. 35B40. 35K55. 92A90

Key words and phrases. Travelling wave, stability, eigenvalue, winding number.

${ }^{1}$ Supported in part by NSF grant \# MCS 8200392. 
These equations were originally formulated as a simplification to the HodgkinHuxley equations for nerve conduction; see FitzHugh [14] and Nagumo et al. [21]. They have since become a central example in reaction-diffusion equations.

A solution to (1.1) is determined by an initial value

$$
u(x, 0)=u_{0}(x), \quad w(x, 0)=w_{0}(x),
$$

where $x$ ranges over $\mathbf{R}$. In the nerve conduction case the variable $x$ is the distance along the nerve fiber.

The initial value problem (1.1), (1.3) can be solved (at least for small time) in many different function spaces; see Rauch and Smoller [22]. A natural one for our purposes is the space

$$
\mathrm{BC}\left(\mathbf{R}, \mathbf{R}^{2}\right)=\left\{u: \mathbf{R} \rightarrow \mathbf{R}^{2} \mid u \text { is bounded and uniformly continuous }\right\}
$$

supplied with the supremum norm.

A travelling wave for (1.1) is a solution that is a function of the single variable $\xi=x-c t$, i.e., $(u(\xi), w(\xi))$ satisfies

$$
-c u^{\prime}=u^{\prime \prime}+f(u)-w, \quad-c w^{\prime}=\varepsilon(u-\gamma w) \quad\left({ }^{\prime}=d / d \xi\right) .
$$

A travelling pulse is a travelling wave that satisfies $(u, w) \rightarrow(0,0)$ as $\xi \rightarrow \pm \infty$.

For the nerve conduction problem, $(0,0)$ is the rest state and the nerve impulse is such a travelling wave.

The existence of a relevant travelling pulse, for some value of $c$, has been proved by many authors for $\varepsilon \ll 1$; see Carpenter [2], Conley [3], Hastings [17] and Langer [20]. Whether there exists such a pulse for $\varepsilon$ not necessarily small is an open question. The significance of $\varepsilon$ small is that (1.4) then becomes a singular perturbation and the pulse is constructed by piecing together solutions of certain reduced systems. The most explicit construction is given by Langer [20].

Call this travelling pulse $\left(u_{\varepsilon}(\xi), w_{\varepsilon}(\xi)\right)$. I shall be interested in its stability relative to the original PDE (1.1). If (1.1) is recast in a moving coordinate frame, i.e., in terms of variables $\xi=x-c t$ and $t$, it becomes

$$
u_{t}=u_{\xi \xi}+c u_{\xi}+f(u)-w, \quad w_{t}=c w_{\xi}+\varepsilon(u-\gamma w) .
$$

The travelling wave is an equilibrium (time independent) solution of (1.5). The fact that any translate of a travelling wave is also a travelling wave must be taken into account when defining stability. In the following:

$$
U=(u, w) \text { and } U_{\varepsilon}(\xi)=\left(u_{\varepsilon}(\xi), w_{\varepsilon}(\xi)\right) .
$$

Definition. The travelling wave, for fixed $\varepsilon>0$, is said to be stable if there exists $\delta>0$ so that if $U(\xi, t)$ is a solution of $(1.5)$ and there is a $k_{1}$ so that $\| U\left(\xi+k_{1}, 0\right)-$ $U_{\varepsilon}(\xi) \|_{\infty}<\delta$, then there is a $k_{2}$ such that

$$
\left\|U\left(\xi+k_{2}, t\right)-U_{\varepsilon}(\xi)\right\|_{\infty} \rightarrow 0
$$

as $t \rightarrow+\infty$.

This says that if a solution to (1.5) starts near some translate of the travelling wave, it tends to some other translate of it as $t \rightarrow+\infty$. A standard technique for determining stability is to use the linearised criterion. If the right-hand side of (1.5) 
is linearised about its equilibrium solution $U_{\varepsilon}(\xi)$, the resulting operator is

$$
L\left(\begin{array}{l}
p \\
r
\end{array}\right)=\left(\begin{array}{c}
p_{\xi \xi}+c p_{\xi}+f^{\prime}\left(u_{\varepsilon}\right) p-r \\
c r_{\xi}+\varepsilon(p-\gamma r)
\end{array}\right),
$$

where

$$
\left(\begin{array}{l}
p \\
r
\end{array}\right)(\xi) \in \mathrm{BC}\left(\mathbf{R}, \mathbf{R}^{2}\right)
$$

The linearised criterion for stability of the travelling pulse is that the spectrum of $L$ (except for 0 ) lies in a left half-plane $\{\lambda: \operatorname{Re} \lambda<a\}$ where $a<0$, and 0 is a simple eigenvalue. Note that 0 must be in the spectrum because the translate of a travelling wave is another travelling wave. 0 being a simple eigenvalue means that this is the only neutral effect. This paper is devoted to proving

THEOREM. Let $L$ be given by (1.7), L: BC $\rightarrow \mathrm{BC}$. Then

(1) there exists $a<0$ so that $\sigma(L) \backslash\{0\} \subset\{\lambda: \operatorname{Re} \lambda<a\}$;

(2) 0 is a simple eigenvalue.

Whether linearised stability implies stability relative to the full (nonlinear) equations, in the sense of the definition above, is a separate question. Henry [18] has some general theorems but these require a sectorial operator, and $L$ is not sectorial as it has some spectrum that is asymptotically vertical; see $\$ 3$.

In [8] Evans proved a "linearised stability implies stability" theorem for "nerve impulse equations". This is a class of equations that includes the FitzHugh-Nagumo system with the stated parameter values. The theorem in [8], in fact, states that the linear PDE is stable if the above described conditions on the spectrum hold. There is then a result in [6] which states that the travelling wave is stable for the full PDE. Using this, the following can be concluded from the theorem.

COROLlaRY. If $\varepsilon \ll 1, U_{\varepsilon}(\xi)$ is stable in the sense of the definition.

In the next section the construction of the travelling pulse solution, found by the authors mentioned, is sketched. A theorem is then proved that gives an exact description of the fact that the pulse approaches the singular orbit as $\varepsilon \rightarrow 0$.

The spectrum of $L$ falls naturally into two pieces: the normal spectrum, consisting of eigenvalues of finite multiplicity; and the essential spectrum, which is the rest. It is shown in $\$ 3$ that the essential spectrum lies in a half-plane $\{\lambda: \operatorname{Re} \lambda<a\}$ for some $a<0$. This essentially follows from proving that the system (1.1) is stable at $(0,0)$, which is an assumption Evans makes for the theorem referenced above from [8].

In the set $\{\lambda: \operatorname{Re} \lambda>a\}$ an analytic function, due to Evans, $D(\lambda)$ can be defined. The zeroes of $D(\lambda)$ are eigenvalues of $L$. The description of $D(\lambda)$ is also given in $\S 3$.

$D(\lambda)$ is used to approximately locate the eigenvalues of $L$. They must lie close to the eigenvalues for a certain reduced system that is associated with some pieces in the singular travelling wave $(\varepsilon=0)$.

The reduced system is analysed in $\$ 4$ and this approximate location of the eigenvalues of the full system is proved in $\$ 5$. 
It then follows that the only danger to stability comes from eigenvalues that lie near zero. In \$6 I prove that there are at most two eigenvalues near zero. This is a computation of the winding number of $D$ applied to a small circle about 0 (actually, it is not $D$, but an analytic continuation $\tilde{D}$ ). Since $D$ is analytic, this winding number measures the number of zeroes inside the circle. It is proved that this winding number is exactly 2 .

Zero is of necessity an eigenvalue, due to translation of the waves. Therefore, the other eigenvalue is real. In $\$ 7$ the proof is completed by showing that this other eigenvalue is negative. Evans derived a very beautiful technique for determining this kind of information. He showed that the sign of the quantity $\left.(d / d \lambda) D(\lambda)\right|_{\lambda=0}$ is determined by the direction in which the stable and unstable manifolds cross in the construction of the pulse. This is determined by using Langer's construction of the pulse.

ACKnowledgement. I am very grateful to R. Pego for pointing out the incorrectness of the proof in $\S 7$ of an earlier version of this manuscript. I am also grateful to him for making very helpful suggestions as to how to correct it.

I am very grateful to Professors C. Conley, J. Evans, N. Fenichel, P. Fife and D. Terman for sharing with me some of their insights on this and related problems.

2. Description of the pulse. The travelling pulse satisfies (1.4), rewritten as a system

$$
u^{\prime}=v, \quad v^{\prime}=-c v-f(u)+w, \quad w^{\prime}=-(\varepsilon / c)(u-\gamma w) .
$$

The phase space of $(2.1)$ is $\mathbf{R}^{3}$. The origin $(0,0,0)$ is a critical point of $(2.1)$ and the pulse solution is a homoclinic orbit to the origin.

This homoclinic orbit is constructed for $\varepsilon \ll 1$. Langer describes the limiting behavior of this orbit, as $\varepsilon \rightarrow 0$, in some detail in his $\$ 2$. I shall review this description, using his notation as much as possible.

When $\varepsilon=0$ each plane $w=$ constant is invariant for (2.1). There exist values $w_{\max }$ and $w_{\min }$, with $w_{\min }<0$, so that if $w_{\min }<w<w_{\max }$ then the reduced system

$$
u^{\prime}=v, \quad v^{\prime}=-c v-f(u)+w
$$

has three critical points. When $w=0$ there is a $c^{*}<0$ for which there exists a heteroclinic orbit, called $J_{F}$, joining $(0,0,0)$ to the right-most critical point $(1,0,0)$. For $c^{*}$ there is a $w^{*}$ for which an orbit, called $J_{\mathrm{B}}$, exists to (2.2) joining the right to the left critical point. F and B stand for front or back; an explanation for this will be given after the pulse is described further.

The singular limit of the homoclinic orbit $(\varepsilon \rightarrow 0)$ consists of four pieces:

(1) $J_{\mathrm{F}}$;

(2) $E_{\mathrm{R}}^{*}=\left\{(u, v, w): v=0,0 \leqslant w \leqslant w^{*}\right.$ and $u$ is the largest root of $\left.w=f(u)\right\}$;

(3) $J_{\mathrm{B}}$;

(4) $E_{\mathrm{L}}^{*}=\left\{(u, v, w): v=0,0 \leqslant w \leqslant w^{*}\right.$ and $u$ is the smallest root of $\left.w=f(u)\right\}$; see Figure 1 .

Let $S_{0}=J_{\mathrm{F}} \cup E_{\mathrm{R}}^{*} \cup J_{\mathrm{B}} \cup E_{\mathrm{L}}^{*} \subset \mathbf{R}^{3} . S_{0}$ is the singular orbit. It is called singular because $E_{\mathrm{R}}^{*}$ and $E_{\mathrm{L}}^{*}$ consist of critical points. The existence theorem says that, given 
any neighborhood $N$ of $S_{0}$, there is an $\varepsilon_{0}$ so that (2.1) has a solution for some $c=c(\varepsilon)$ for all $\varepsilon \in\left[0, \varepsilon_{0}\right]$, which is homoclinic to $(0,0,0)$ and lies entirely in $N$. Moreover, $c(\varepsilon) \rightarrow c^{*}$ as $\varepsilon \rightarrow 0$. Call this orbit of $(2.1), S_{\varepsilon}$.

This picture is not new to Langer's proof but was already in the earlier proofs. Langer's contribution was to add that if $N$ is a small enough neighborhood of $S_{0}$, there is a unique solution for each $\varepsilon$ for unique $c$.

Langer uses a transversality argument. He shows that two certain manifolds intersect transversely in $(u, v, w, c)$-space for $\varepsilon=0$; therefore, they still intersect for $\varepsilon$ small. The uniqueness follows from the transversality. For the stability proof, some information about the nature of this transversality will play a central role; see $\S 7$.

If the pulse solution is graphed with $U$ as a function of $\xi$, a profile is obtained that looks like a nerve impulse but with a long latent period in the middle. The part close to $J_{\mathrm{F}}$ is the front and that close to $J_{\mathrm{B}}$ is the back.

I shall need a more explicit description of $S_{\varepsilon}$. This is contained in the following theorem.

THEOREM 2.1. If $\varepsilon_{0}$ is sufficiently small, there exists a homeomorphism $h: S^{1} \times$ $\left[0, \varepsilon_{0}\right] \rightarrow \cup S_{\varepsilon}$, where the union is taken over $\varepsilon \in\left[0, \varepsilon_{0}\right]$.

Proof. Firstly, parametrise $S_{0}$ in any way, i.e., choose a map $h_{0}: S^{1} \rightarrow S_{0}$. I shall show this can be extended.

Let $U_{0}, U_{1}, U_{2}, U_{3}$ denote the four corners of $S_{0}$; see Figure 2. Define $B \subset \mathbf{R}^{3}$ by

$$
B=\left[-\gamma_{1}, \gamma_{1}\right] \times\left[-\gamma_{2}, \gamma_{2}\right] \times\left[-\gamma_{3}, \gamma_{3}\right]
$$

Let $B_{i}=U_{i}+B$; see Figure 2 . Choose the $\gamma_{i}$ linearly related so that $J_{\mathrm{F}}$ and $J_{\mathrm{B}}$ cross $\partial B_{i}$ through faces parallel to the $u=0$ plane and $E_{\mathrm{R}}^{*}, E_{\mathrm{L}}^{*}$ cross through faces parallel to $w=0$.

Let $b_{i}, i=1, \ldots, 8$, be the successive intersection points of $\partial\left(B_{0} \cup B_{1} \cup B_{2} \cup B_{3}\right)$ with $S_{0}$ starting at $J_{F} \cap B_{0}$ and proceeding in a counterclockwise direction. Set

$$
M_{\mathrm{F}}=\left\{(u, v, w): u=0 \text { and } v^{2}+w^{2} \leqslant \gamma_{\mathrm{F}}\right\} \text {. }
$$

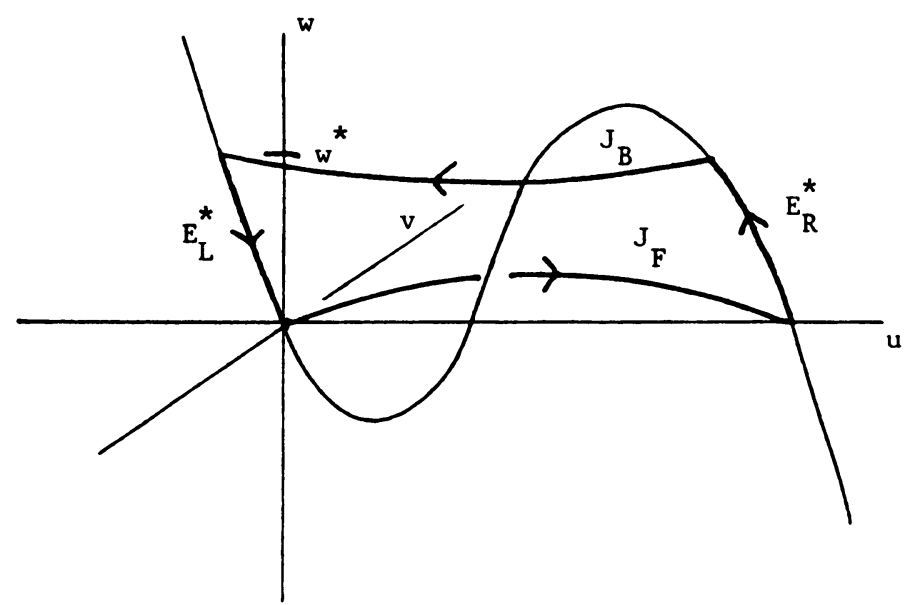

Figure 1 
Now choose $\gamma_{\mathrm{F}}$ so that $b_{1}+M_{\mathrm{F}} \subset \partial B_{0}$ and $b_{2}+M_{\mathrm{F}} \subset \partial B_{1}$. Similarly, set

$$
M_{\mathrm{R}}=\left\{(u, v, w): w=0 \text { and } u^{2}+v^{2} \leqslant \gamma_{\mathrm{R}}\right\} .
$$

Choose $\gamma_{\mathrm{R}}$ so that $b_{3}+M_{\mathrm{R}} \subset \partial B_{1}$ and $b_{4}+M_{\mathrm{R}} \subset \partial B_{2}$. Define $M_{\mathrm{B}}$ and $M_{\mathrm{L}}$ similarly to $M_{\mathrm{F}}$ and $M_{\mathrm{R}}$, respectively; again choose $\gamma_{\mathrm{B}}$ and $\gamma_{\mathrm{L}}$ so that the obvious conditions are satisfied.

Let $\tilde{J}_{\mathrm{F}}=J_{\mathrm{F}} \backslash\left\{B_{0} \cup B_{1}\right\}$; form a tube about $\tilde{J}_{\mathrm{F}}$ by setting

$$
N_{\mathrm{F}}=\bigcup_{j \in \tilde{J}_{\mathrm{F}}}\left(j+M_{\mathrm{F}}\right)
$$

Define $N_{\mathrm{R}}, N_{\mathrm{B}}$ and $N_{\mathrm{L}}$ in the obvious fashion. Let

$$
N=B_{0} \cup N_{\mathrm{F}} \cup B_{1} \cup N_{\mathrm{R}} \cup B_{2} \cup N_{\mathrm{B}} \cup B_{3} \cup N_{\mathrm{L}} .
$$

$N$ is a neighborhood of $S_{0}$ formed out of tubes joining boxes that cover each corner.

The size of the neighborhood is determined by $\gamma_{1}$, say, since each of the other $\gamma$ 's is related to it. Let $\kappa=\gamma_{1}$; then $N=N(\kappa)$ and, as $\kappa \rightarrow 0, N \rightarrow S_{0}$ as a set. Consequently, for fixed $\kappa$, there is an $\varepsilon_{0}>0$ so that $S_{\varepsilon} \subset N$ for all $\varepsilon \in\left[0, \varepsilon_{0}\right]$.

By the chosen parametrisation of $S_{0}, h \mid S^{1} \times\{0\}=h_{0}$ is already defined. Now I shall extend $h_{0}$ to $S^{1} \times\left[0, \varepsilon_{0}\right]$. Let $(\theta, \varepsilon) \in S^{1} \times\left[0, \varepsilon_{0}\right]$; there are two cases to consider.

Case I. $h_{0}(\theta) \notin B_{i}$ for any $i$. Then $h_{0}(\theta) \in N_{\mathrm{F}} \cup N_{\mathrm{R}} \cup N_{\mathrm{B}} \cup N_{\mathrm{L}}$. Let $M_{\theta}=$ $h_{0}(\theta)+M_{F}$ and set $h(\theta, \varepsilon)=S_{\varepsilon} \cap M_{\theta}$.

A priori, the right-hand side is just a set. But from the equation $u^{\prime}=v$ in (2.1) it is clear that it contains just one point and so the map is well defined.

Case II. $h_{0}(\theta) \in B_{i}$. Consider $B_{1}$; the others are analogous. Form a rectangle $M_{\theta}$ in $\mathbf{R}^{3}$ as follows. Let $P_{\theta}=$ plane containing $h_{0}(\theta)$ and the line $u=u_{1}-\gamma_{1}, w=\gamma_{3}$, where $U_{1}=\left(u_{1}, v_{1}, w_{1}\right)$ is the corner point. Let $M_{\theta}=P_{\theta} \cap B_{1}$. Define $h(\theta, \varepsilon)=S_{\varepsilon}$ $\cap \boldsymbol{M}_{\boldsymbol{\theta}}$.

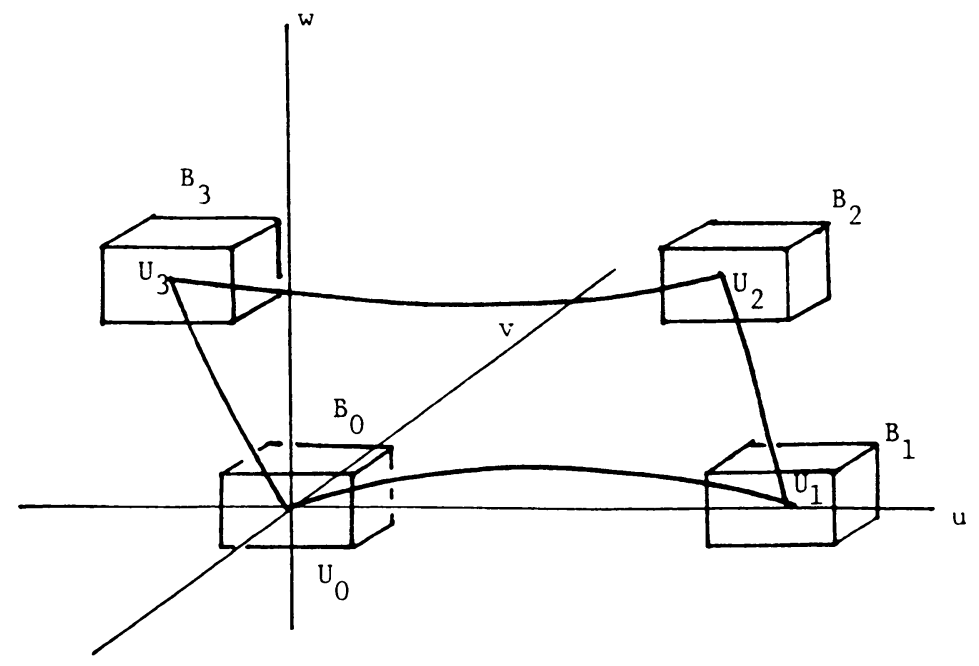

Figure 2 
It is considerably harder to see that $h$ is well defined in this case due to the subtlety of the behavior of the wave near the corners. $B_{0}$ is actually straightforward because of the approximation of the stable and unstable manifolds by the eigenspaces.

Let $h_{0}\left(\bar{\theta}_{1}\right)=b_{2}$ and $h_{0}\left(\bar{\theta}_{2}\right)=b_{3}$; these are the entrance and exit points of $S_{0}$ through $B_{1}$. I shall prove the following lemma.

LEMMA 2.1. If $\kappa$ is sufficiently small (and consequently $\varepsilon_{0}$ ), $S_{\varepsilon} \cap M_{\theta}$ contains a unique point for $\bar{\theta}_{1} \leqslant \theta \leqslant \bar{\theta}_{2}$.

Proof. I shall divide this into two cases. Choose $\bar{\theta}$ so that $h_{0}(\bar{\theta}) \in J_{\mathrm{F}} \cap B_{1}$ but $\bar{\theta} \neq \bar{\theta}_{1}$ and $h_{0}(\bar{\theta}) \neq U_{1}$. Let $m_{\theta}=$ slope of $M_{\theta}$ projected onto $(u, w)$ space; see Figure 3. Reset $\gamma_{1}$ and $\gamma_{3}$, if necessary, so that $m_{\bar{\theta}}>f^{\prime}(0)+\delta$ for some $\delta>0$.

Let $n_{\theta}=$ normal to $M_{\theta}$ with a positive $u$ component. It suffices to show that

$$
n_{\theta} \cdot\left(u_{\varepsilon}^{\prime}(\xi), v_{\varepsilon}^{\prime}(\xi), w_{\varepsilon}^{\prime}(\xi)\right)>0
$$

for any $\theta \in\left[\bar{\theta}_{1}, \bar{\theta}_{2}\right]$ such that $\left(u_{\varepsilon}(\xi), v_{\varepsilon}(\xi), w_{\varepsilon}(\xi)\right) \in M_{\theta}$.

Case I. $\theta \in\left[\bar{\theta}_{1}, \bar{\theta}\right]$. Suppose (2.3) were violated for all $\kappa>0$ with some $\theta$ in $\left[\bar{\theta}_{1}, \bar{\theta}\right]$; then there would be a sequence of points on $S_{\varepsilon}$ as $\varepsilon \rightarrow 0$ for which (2.3) failed. These would converge to a point $h_{0}(\theta)$ on $S_{0}$. In fact, $h_{0}(\theta) \in J_{\mathrm{F}}$ and, by continuity of the vector field (call it $V$ ),

$$
n_{\theta} \cdot V\left(h_{0}(\theta)\right) \leqslant 0 .
$$

Since $h_{0}(\theta) \in J_{\mathrm{F}}$ this is impossible unless $h_{0}(\theta)=U_{1}$, but it cannot be in Case I.

Case II. $\theta \in\left[\bar{\theta}, \bar{\theta}_{2}\right]$. To obtain information about the derivative along $S_{\varepsilon}$, consider the variational equations

(2.4) $\delta u^{\prime}=\delta v, \quad \delta v^{\prime}=-c \delta v-f^{\prime}\left(u_{\varepsilon}\right) \delta u+\delta w, \quad \delta w^{\prime}=-(\varepsilon / c)(\delta u-\gamma \delta w)$.

If $\varepsilon$ is small and $U_{\varepsilon} \in B_{1},(2.4)$ is well approximated by the system linearised at $U_{1}$ with $\varepsilon=0$;

$$
\delta u^{\prime}=\delta v, \quad \delta v^{\prime}=-c \delta v-f^{\prime}\left(u_{1}\right) \delta u+\delta w, \quad \delta w^{\prime}=0 .
$$

Because they are linear, both (2.4) and (2.5) induce flows on $S^{2}$ by equating two vectors in $\mathbf{R}^{3} \backslash\{0\}$ if one is a positive multiple of the other. The flow of (2.5) is

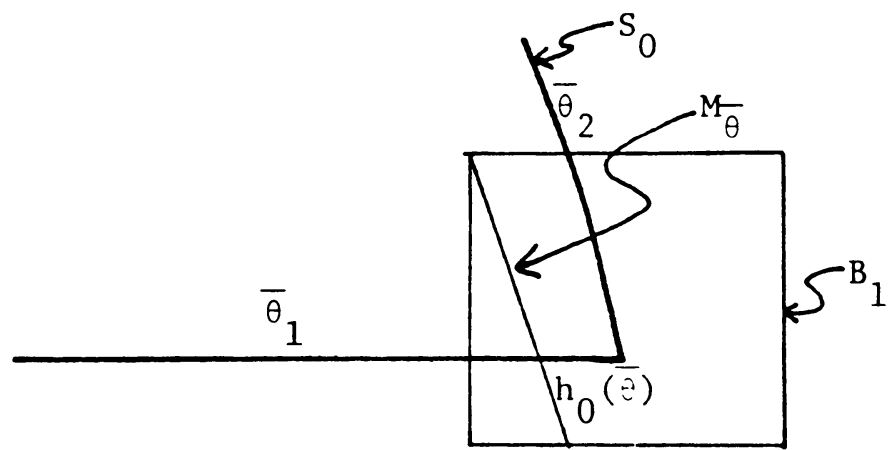

Figure 3 
qualitatively the same as the linearisation at rest. It has one unstable subspace and two stable ones. Let these be spans of the eigenvectors $X_{1}$ (unstable), $X_{2}$ and $X_{3}$.

The associated flow on $S^{2}$ has two attracting critical points, two repelling ones, and two saddles; see Figure 4. These come from the eigenspaces. Let $X_{2}$ be the eigenvector that gives the saddle. Set $C=\operatorname{span}\left\{X_{2}, X_{3}\right\} \cap S^{2}$ and let $V$ be a given neighborhood of $C$ in $S^{2}$.

If $\tilde{U}_{\varepsilon}(\xi)=U_{\varepsilon}^{\prime}(\xi) /\left|U_{\varepsilon}^{\prime}(\xi)\right|$, this satisfies the flow induced on $S^{2}$ from (2.4). If $\varepsilon_{0}$ is small enough, $\tilde{U}_{\varepsilon}(\xi) \in V$, while $U_{\varepsilon}(\xi) \in B_{1}$; otherwise, it would be driven to some neighborhood of $\operatorname{span}\left\{X_{1}\right\} \cap S^{2}$, since these two points are attractors for the flow on $S^{2}$ derived from (2.5). If this happened, $U_{\varepsilon}(\xi)$ would leave $B_{1}$ other than through the top, which it does not.

Consider the vector $\left(u_{\varepsilon}^{\prime}(\xi), w_{\varepsilon}^{\prime}(\xi)\right)$. Since $w_{\varepsilon}^{\prime}(\xi)>0$, if (2.3) were violated it is easy to check that $0>w_{\varepsilon}^{\prime}(\xi) / u_{\varepsilon}^{\prime}(\xi)>f^{\prime}(0)+\delta$ for $\theta \in\left[\bar{\theta}_{\bar{\theta}} \bar{\theta}_{2}\right]$. But this is impossible if $\varepsilon_{0}$ is small enough, because (2.5) is then well approximated by (2.4) and, inside $C$, $\operatorname{span}\left\{X_{1}\right\} \cap S^{2}$ is a pair of attracting points. Moreover, $X_{1}=\left(-1,0,-f^{\prime}\left(u_{1}\right)\right)$. This completes the proof of the lemma.

Returning to the proof of Theorem 2.1, it is now known that $h(\theta, \varepsilon)$ is well defined. It is obviously one-to-one, since the $M_{\theta}$ 's are all disjoint. Because $S^{1} \times\left[0, \varepsilon_{0}\right]$ is compact, it remains to show that $h$ is continuous. By Langer's proof, since it uses the implicit function theorem, $h$ is continuous in $\varepsilon$ for each $\theta$. By continuity of the flow this is uniform in $\theta$; full continuity therefore follows.

3. Essential spectrum and the definition of $D(\lambda)$. Firstly, I shall give the definitions used in splitting up the spectrum. Let $B$ be a Banach space and $L: B \rightarrow B$ a linear operator.

Definition. $\lambda \in \mathbf{C}$ is said to be in the normal spectrum, denoted $\sigma_{n}(L)$, if it is an isolated eigenvalue of finite multiplicity.

The essential spectrum, $\sigma_{\mathrm{e}}(L)$, is the complement of this in $\sigma(L)$, i.e., $\sigma_{\mathrm{e}}(L)=$ $\sigma(L) \backslash \sigma_{\mathrm{n}}(L)$.

Now let $L$ be the linearised operator about the travelling wave given by (1.7) and let $B=\mathrm{BC}\left(\mathbf{R}, \mathbf{R}^{2}\right)$. In this section I shall prove that $\sigma_{\mathrm{e}}(L)$ is bounded away from the imaginary axis in the left half-plane. Also I shall define Evans' analytic function $D(\lambda)$, which is the tool for finding eigenvalues.

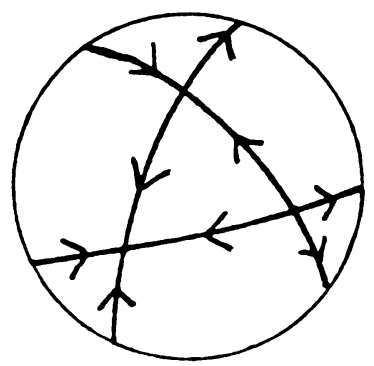

FIGURE 4 
Consider the equation

$$
(L-\lambda I)\left(\begin{array}{l}
p \\
r
\end{array}\right)=0
$$

where $\left(\begin{array}{l}p \\ r\end{array}\right)(\xi) \in B_{c}$, complexified $B$. Rewrite (3.1) as a system

$$
\begin{aligned}
& p^{\prime}=q, \\
& q^{\prime}=-c q+\left(\lambda-f^{\prime}(u)\right) p+r, \\
& r^{\prime}=-(\varepsilon / c) p+((\lambda+\varepsilon \gamma) / c) r .
\end{aligned}
$$

I have dropped the $\varepsilon$ on $U_{\varepsilon}$, so with a slight abuse of notation, $U(\xi)=$ $(u(\xi), v(\xi), w(\xi))$ is the underlying travelling wave.

Let $z=(p, q, r) \in \mathbf{C}^{3}$ and write (3.2) as

$$
z^{\prime}=A z,
$$

where

$$
A=\left(\begin{array}{ccc}
0 & 1 & 0 \\
\lambda-f^{\prime}(u) & -c & 1 \\
-\varepsilon / c & 0 & (\lambda+\varepsilon \gamma) / c
\end{array}\right)
$$

Equation (3.3) is a nonautonomous one on $\mathbf{C}^{3}$. As $\boldsymbol{\xi} \rightarrow \pm \infty, u(\xi) \rightarrow 0$; therefore (3.3) is asymptotically autonomous and the asymptotic system is

$$
z^{\prime}=A_{0} z
$$

where

$$
A_{0}=\left(\begin{array}{ccc}
0 & 1 & 0 \\
\lambda-f^{\prime}(0) & -c & 1 \\
-\varepsilon / c & 0 & (\lambda+\varepsilon \gamma) / c
\end{array}\right) .
$$

The set $S=\left\{\lambda \in \mathrm{C}: A_{0}=A_{0}(\lambda)\right.$ has an imaginary eigenvalue $\}$ will determine the necessary information about $\sigma_{\mathrm{e}}(L)$.

LEMMA 3.1. If $\varepsilon>0, \mathrm{C} \backslash S$ has a component $G$ for which there exists an $a<0$ such that $\{\lambda: \operatorname{Re} \lambda>a\} \subset G$.

Proof. Let $P=P(\alpha, \varepsilon, \lambda)=\operatorname{det}\left(A_{0}-\alpha I\right)$. Then

$$
P=\left(\alpha^{2}+c \alpha+f^{\prime}(0)-\lambda\right)((\lambda+\varepsilon \gamma) / c-\alpha)-\varepsilon / c .
$$

Fix $\varepsilon>0$. $S$ consists of those $\lambda$ for which

$$
P(\alpha, \varepsilon, \lambda)=0
$$

for some $\alpha \in i \mathbf{R}$. If $\varepsilon=0$, the set of $\lambda$ 's for which $P(i \tau, 0, \lambda)=0$, for some $\tau \in \mathbf{R}$, is easily seen to be the imaginary axis union the parabola $\operatorname{Re} \lambda=-(\operatorname{Im} \lambda)^{2} / c^{2}+f^{\prime}(0)$.

For (3.8) the solutions $\lambda$ will be near this curve and near the imaginary axis. The latter are the only ones to worry about. For fixed $\tau$, at $\varepsilon=0$,

$$
\frac{d \lambda}{d \varepsilon}=-\frac{\partial P}{\partial \varepsilon} / \frac{\partial P}{\partial \lambda} \text {. }
$$

Now

$$
\partial P / \partial \lambda=\left(-\tau^{2}+f^{\prime}(0)\right) / c
$$


since $f^{\prime}(0)<0, \partial P / \partial \lambda \neq 0$, and $\lambda$ is a function of $\varepsilon$ for fixed $\alpha=i \tau$ near $\varepsilon=0$ such that $\lambda(0) \in i \mathbf{R}$. For each fixed $\tau$ this gives all $\lambda$ 's for which $A_{0}(\lambda)$ has an imaginary eigenvalue because (3.7) is quadratic in $\lambda$.

Since

$$
d \lambda / d \varepsilon=-\left(\gamma+1 /\left(\tau^{2}-f^{\prime}(0)\right)\right)<0,
$$

the set of $\lambda$ 's near the imaginary axis lies in the left-hand plane. If $\gamma>0$ is fixed, the curve thus defined is bounded uniformly away from the imaginary axis. This proves the lemma.

The point of this lemma is that there is no essential spectrum of $L$ in $G$. Evans shows this for his more general class of problems in Theorem 3 of [8]. The idea is fairly standard and worth explaining briefly.

Set $L=L_{0}+R$, where

$$
L_{0}\left(\begin{array}{l}
p \\
r
\end{array}\right)=\left(\begin{array}{c}
p^{\prime \prime}+c p^{\prime}+f^{\prime}(0) p+r \\
c p^{\prime}+\varepsilon(p-\gamma r)
\end{array}\right)
$$

and

$$
R\left(\begin{array}{l}
p \\
r
\end{array}\right)=\left(\begin{array}{c}
\left(f^{\prime}(u)-f^{\prime}(0)\right) p \\
0
\end{array}\right)
$$

$L_{0}$ is the linearisation about the rest state and $R$ is the perturbation due to the wave. $\sigma\left(L_{0}\right)$ has actually been found in Lemma 3.1. The equation $\left(L_{0}-\lambda I\right)\left({ }_{r}^{p}\right)=0$ becomes $z^{\prime}=A_{0} z$ when rewritten as a system.

It is a standard computation to see that $\sigma\left(L_{0}\right)=S . R$ is a relatively compact perturbation of $L_{0}$. It follows that any component of $C \backslash S$ is entirely the essential spectrum, or the only spectrum in it is normal. Evans further shows that if $\lambda<0$ and large, it is not an eigenvalue. It follows that the only spectrum in $G$ is normal. This kind of argument establishes the following lemma.

LEMMA 3.2. $\sigma(L) \cap G \subset \sigma_{\mathrm{n}}(L)$.

REMARKs. (1) $a=a(\varepsilon)$ and tends to 0 as $\varepsilon \rightarrow 0$, so there is not a right half-plane whose boundary is bounded to the left of the imaginary axis independently of $\varepsilon$.

(2) $S$ contains a curve that is asymptotically vertical, thus preventing $L$ and $L_{0}$ from being sectorial.

Lemmas 3.1 and 3.2 show that $\sigma_{\mathrm{e}}(L)$ causes no problem for stability. Hence, I need only be concerned with locating eigenvalues. As stated earlier, this is done by defining an analytic function $D(\lambda)$ whose domain is $G$.

Consider again $A_{0}$, given by (3.6). I claim that if $\lambda \in G, A_{0}(\lambda)$ has only one eigenvalue of positive real part. It is easy to check this for $\varepsilon=0$ from the definition of $G$. It therefore follows for $\varepsilon>0$. Call this eigenvalue $\alpha^{+}=\alpha^{+}(\lambda, \varepsilon)$. Its associated eigenvector can be written

$$
X^{+}=\left(1, \alpha^{+},-\varepsilon /\left[c \alpha^{+}-(\lambda+\varepsilon \gamma)\right]\right) .
$$

Since $P(\alpha, \varepsilon, \lambda)=0$ simplifies as $\varepsilon \rightarrow 0, \alpha^{+}$can be given explicitly in the limit

$$
\alpha^{+}(\lambda, 0)=\left(-c+\left(c^{2}-4\left(f^{\prime}(0)-\lambda\right)\right)^{1 / 2}\right) / 2 .
$$


In the following, assume $\varepsilon \neq 0$. I shall motivate the definition of $D(\lambda)$ by seeing what it means to look for an eigenvalue. An eigenvalue of $L$ in $G$ is a $\lambda$ for which there is a solution of (3.2) that is bounded at $\pm \infty$. For it to be bounded at $-\infty$, it must be asymptotic to the unstable eigenspace.

By Evans [9] there is a unique solution $\zeta(\lambda, \xi)$ to (3.3) that satisfies

$$
\zeta(\lambda, \xi)-X^{+} e^{\alpha^{+} \xi} \rightarrow 0
$$

as $\xi \rightarrow-\infty$ faster than $e^{\operatorname{Re} \alpha^{+} \xi}$. Furthermore, $\zeta(\lambda, \xi)$ is a $C^{3}$-valued analytic function of $\lambda \in G$ for each fixed $\xi$.

This function $\zeta(\lambda, \xi)$ is therefore a candidate to be an eigenfunction and, up to a scalar multiple, it is the only one.

To see if it is bounded at $+\infty$, one uses the adjoint to (3.3),

$$
z^{* \prime}=B z^{*} \text {, }
$$

where $B=-A^{*}$, so

$$
B=\left(\begin{array}{ccc}
0 & f^{\prime}(u)-\bar{\lambda} & \varepsilon / c \\
-1 & c & 0 \\
0 & -1 & -(\bar{\lambda}+\varepsilon \gamma) / c
\end{array}\right)
$$

The asymptotic system for $(3.10)$ is

$$
z^{* \prime}=B_{0} z^{*}
$$

where $B_{0}$ is the same as $B$ but with $u$ replaced by 0 . Obviously $B_{0}=-A_{0}^{*}$, and the eigenvalues of $B_{0}$ are the negatives of the complex conjugates of the eigenvalues of $A_{0} . B_{0}$ therefore has a unique eigenvalue of negative real part in $G$; call it $\beta^{-}=\beta^{-}(\lambda, \varepsilon)=-\bar{\alpha}^{+}$. Its associated eigenvector is

$$
Y^{-}=\left(1,\left(c-\beta^{-}\right)^{-1},\left[\left(\beta^{-}-c\right)\left(\beta^{-}+(\bar{\lambda}+\varepsilon \gamma) / c\right)\right]^{-1}\right) .
$$

(3.10) therefore has a unique solution $\eta(\lambda, \xi)$ satisfying

$$
\eta(\lambda, \xi)-Y^{-} e^{\beta^{-} \xi} \rightarrow 0
$$

as $\xi \rightarrow+\infty$ faster than $e^{\operatorname{Re} \beta^{-} \xi}$. Furthermore, $\eta(\lambda, \xi)$ is a $C^{3}$-valued analytic function of $\lambda$ for each fixed $\xi$.

Definition. The function $D(\lambda)=\zeta(\lambda, \xi) \cdot \eta(\lambda, \xi)$.

One checks easily that this is well defined, i.e. independent of $\xi$ :

$$
\begin{aligned}
\frac{\partial}{\partial \xi} D(\lambda) & =\frac{\partial}{\partial \xi} \zeta(\lambda, \xi) \cdot \eta(\lambda, \xi)+\zeta(\lambda, \xi) \cdot \frac{\partial}{\partial \xi} \eta(\lambda, \xi) \\
& =A \zeta \cdot \eta+\zeta \cdot B \eta=A \zeta \cdot \eta-\zeta \cdot A^{*} \eta \\
& =0 .
\end{aligned}
$$

I shall collect the important properties of $D(\lambda)$.

Properties of $D(\lambda)$. (1) $D: G \rightarrow C$ is analytic.

(2) Zeroes of $D(\lambda)$ are eigenvalues of $L$.

(3) The order of a zero is equal to the algebraic multiplicity of the eigenvalue. 
(1) follows from the fact that $\zeta$ and $\bar{\eta}$ are analytic functions of $\lambda$, into $\mathbf{C}^{3}$, for each fixed $\xi$. The reason for this can be seen from the proof of Lemma 3.3 below. (2) has a very pretty geometric interpretation. Since (3.3) is linear, its solution operator takes planes to planes (a plane being a two-dimensional complex subspace of $\mathbf{C}^{3}$ ). The information as to how this occurs is contained in the adjoint equation (3.10). In fact, the normal to a plane evolving under (3.3) will satisfy (3.10) if its complex amplitude is determined appropriately. The eigenvector $Y^{-}$is exactly the one that is normal to the stable subspace for (3.5). If $D(\lambda)=0$ then, as $\xi \rightarrow+\infty, \zeta(\lambda, \xi)$ is perpendicular to $Y^{-}$and so is asymptotic to the stable subspace of (3.5). Therefore, $\zeta(\lambda, \xi) \rightarrow 0$ as $\xi \rightarrow+\infty$ and one has an eigenfunction; $\lambda$ is therefore an eigenvalue. It is not hard to see that this is the only way a bounded, uniformly continuous solution of (3.3) can be found. (3) is somewhat more difficult to see and I refer to Evans [9].

I shall need, in $\S 5$, an analytic continuation of $D(\lambda)$ to a right half-plane $\{\lambda$ : $\operatorname{Re} \lambda>b\}$, where $b<0$ and independent of $\varepsilon$. I shall prove this as a lemma which includes the proof of (1).

LEMMA 3.3. There exists $b<0$, independent of $\varepsilon$, and an analytic function $\tilde{D}(\lambda)$ on the set $\tilde{G}=\{\lambda: \operatorname{Re} \lambda>b\}$ so that $\left.\tilde{D}\right|_{G}=D$.

Proof. It will be obvious from the construction that $\tilde{D}$ extends $D$. The problem with $D$ is that the boundary of its domain $G$ collapses onto the imaginary axis as $\varepsilon \rightarrow 0$. The proof is then to produce $\zeta(\lambda, \xi)$ and $\eta(\lambda, \xi)$, satisfying their respective defining conditions. This is possible on a set of the form $\tilde{G}$.

The eigenvalue $\alpha^{+}(\lambda, \varepsilon)$ can be extended to a set of the form $\tilde{G}$ for some $b<0$ independent of $\varepsilon$. This cannot be done preserving the condition that $\alpha^{+}$is the unique eigenvalue of positive real part, but it can be done with $\alpha^{+}$the eigenvalue of largest real part.

In a strip $H=\{\lambda: b<\operatorname{Re} \lambda<0\}$, if $\varepsilon=0$ there are three distinguished eigenvalues:

$$
\begin{gathered}
\alpha^{+}=\left(-c+\left(c^{2}-4\left(f^{\prime}(0)-\lambda\right)\right)^{1 / 2}\right) / 2, \quad \alpha^{0}=\lambda / c, \\
\alpha^{-}=\left(-c-\left(c^{2}-4\left(f^{\prime}(0)-\lambda\right)\right)^{1 / 2}\right) / 2,
\end{gathered}
$$

where a branch of the square root that is continuous near $\arg z=0$ is being used. It is clear that $b$ can be chosen so that $\operatorname{Re} \alpha^{+}>\operatorname{Re} \alpha^{0}>\operatorname{Re} \alpha^{-}$for $\lambda \in H$. One checks easily that if $\varepsilon \ll 1$ and $\lambda \in H$ there are eigenvalues of $A_{0}-\alpha^{+}(\lambda, \varepsilon), \alpha^{0}(\lambda, \varepsilon)$ and $\alpha^{-}(\lambda, \varepsilon)$-corresponding to each of these. Furthermore, $\left|\partial \alpha^{+} / \partial \varepsilon\right|,\left|\partial \alpha^{0} / \partial \varepsilon\right|$ and $\left|\partial \alpha^{-} / \partial \varepsilon\right|$ are bounded independently of $\lambda \in H$. It follows that $\varepsilon>0$ can be chosen so that

$$
\operatorname{Re} \alpha^{+}(\lambda, \varepsilon)>\max \left\{\operatorname{Re} \alpha^{0}(\lambda, \varepsilon), \operatorname{Re} \alpha^{-}(\lambda, \varepsilon)\right\}
$$

for all $\lambda \in H$.

Under these conditions $\zeta(\lambda, \xi)$ can be constructed. The construction of $\eta(\lambda, \xi)$ is analogous with one added difficulty; see comment at end. The construction follows Evans. 
Write (3.3) as

$$
z^{\prime}=A_{0} z+P(\xi) z .
$$

It is easy to check that there exists $C, k>0$, so that

$$
\|P(\xi)\|<C e^{k \xi} \text { for } \xi<0 .
$$

Define the iteration scheme:

$$
\begin{gathered}
\zeta_{0}(\lambda, \xi)=X^{+} e^{\alpha^{+} \xi}, \\
\zeta_{n}(\lambda, \xi)=X^{+} e^{\alpha^{+} \xi}+\int_{-\infty}^{\xi} \exp \left(A_{0}(\xi-s)\right) P(s) \zeta_{n-1}(\lambda, s) d s .
\end{gathered}
$$

That $\zeta_{n}(\lambda, \xi)$ is well defined for $\lambda \in \tilde{G}$ and an analytic function, for fixed $\xi$, is established inductively. The following estimates are shown to hold at the same time. Fix $\lambda_{0} \in \tilde{G}$. There exists a neighborhood $N$ of $\lambda_{0}$ and constants $C_{1}, C_{2}$ independent of $n$ so that for $\xi \leqslant \xi^{*}$, some $\xi^{*}$,

$$
\begin{aligned}
& \text { (1) }\left|\zeta_{n}(\lambda, \xi)\right| \leqslant C_{1} \exp \left(\tau^{-} \xi\right), \\
& \text { (2) }\left|\zeta_{n}(\lambda, \xi)-X^{+} e^{\alpha^{+} \xi}\right| \leqslant C_{2} \exp \left(\tau^{+} \xi\right)
\end{aligned}
$$

where $\tau^{-}=\inf _{\lambda \in N}\left\{\operatorname{Re} \alpha^{+}(\lambda)\right\}$ and $\tau^{+}=\sup _{\lambda \in N}\left\{\operatorname{Re} \alpha^{+}(\lambda)\right\}$. I shall drop mentioning the dependence of $\alpha^{+}$on $\varepsilon$.

The key point is that for $\lambda \in \tilde{G}, \alpha^{+}(\lambda)$ is the eigenvalue of largest real part. Consequently, $\left\|\exp \left(A_{0}(\lambda)\right)\right\| \leqslant \exp \left(\operatorname{Re} \alpha^{+}(\lambda)\right)$.

Choose $N$ so that $\tau_{-}+k>\tau_{+}$. Suppose (1) hold up to $n-1$; then

$$
\begin{aligned}
& \left|\int_{-\infty}^{\xi} \exp \left(A_{0}(\xi-s)\right) P(s) \zeta_{n-1}(\lambda, s) d s\right| \\
& \quad \leqslant C C_{1} \int_{-\infty}^{\xi} \exp \left(\operatorname{Re} \alpha^{+}(\lambda)(\xi-s)+k s+\tau^{-} s\right) d s .
\end{aligned}
$$

This integral converges uniformly for all $\lambda \in N$, which shows that $\zeta_{n}(\lambda, \xi)$ is well defined and analytic in $\lambda \in \tilde{G}$. By setting

$$
C_{2}=C C_{1} /\left(\tau^{-}+k-\tau^{+}\right),
$$

(2) holds. To check (1):

$$
\left|\delta_{n}(\lambda, \xi)\right| \leqslant\left|X^{+}\right| \exp \left(\operatorname{Re} \alpha^{+} \xi\right)+C_{2} \exp \left(\tau^{+} \xi\right) .
$$

As long as $C_{2}$ is chosen larger than $\left|X^{+}\right|, \xi^{*}$ can be picked so that

$$
\left(\left|X^{+}\right|+C_{2} \exp \left(\tau^{+}-\tau^{-}\right) \xi\right) \leqslant C_{2}
$$

for all $\xi \geqslant \xi^{*}$. It is trivial that (1) and (2) are satisfied for $n=0$.

By a very similar inductive argument, $N, \xi^{*}$ and $C_{3}$ can be found so that

$$
\sup _{\lambda \in N}\left|\zeta_{n+1}(\lambda, \xi)-\zeta_{n}(\lambda, \xi)\right| \leqslant \frac{C_{3}}{2^{n}} \exp \left(\tau^{+} \xi\right) \text {. }
$$


It follows that $\zeta_{n}(\lambda, \xi) \rightarrow \zeta(\lambda, \xi)$, for each fixed $\xi$, uniformly on compact subsets of $\tilde{G} . \zeta(\lambda, \xi)$ is therefore analytic and satisfies the integral equation

$$
\zeta(\lambda, \xi)=X^{+} \exp \left(\alpha^{+} \xi\right)+\int_{-\infty}^{\xi} \exp \left(A_{0}(\xi-s)\right) P(s) \zeta(\lambda, s) d s
$$

and therefore satisfies (3.3).

By letting $n \rightarrow \infty$ in (2) above, $\zeta(\lambda, \xi)$ is seen to satisfy the defining condition of $\zeta$ in the set $G$.

The distinguished solution to the adjoint equation, $\eta(\lambda, \xi)$, is constructed in the same way. However, in this case $k \rightarrow 0$ as $\varepsilon \rightarrow 0$. The size of $N$ will therefore depend on $\varepsilon$. For fixed $\varepsilon>0$, the construction goes through and $\eta(\lambda, \xi)$ is analytic in $\tilde{G}$. Obviously $\eta$ does not exist for $\varepsilon=0$, although $\zeta$ does.

$\tilde{D}(\lambda)$ is then set as $\zeta(\lambda, \xi) \cdot \eta(\lambda, \xi)$.

4. Analysis of the reduced system. The zeroes of $D(\lambda)$ (or $\tilde{D})$ will be related to the eigenvalues of the reduced systems, that is, the linearisation of the PDE about the front or the back. I shall redevelop the theory of the preceding section for the reduced system. It is slightly different because the underlying wave is heteroclinic rather than homoclinic. The necessary information about the zeroes of the reduced $D$-function can then be given, as the stability is well understood in these cases; see Fife and McLeod [13].

I shall consider a system which is exactly the one for the front, but the analysis for the back only requires appropriate reinterpretation.

Consider the PDE (in a moving frame with speed $c$ )

$$
u_{t}=u_{\xi \xi}+c u_{\xi}+f(u) \text {, }
$$

where $f(u)$ is given by (1.2). The travelling wave equation is

$$
u^{\prime}=v, \quad v^{\prime}=-c v-f(u) .
$$

As mentioned in $\S 2$, there exists $c^{*}$ so that $(4.2)$ possesses a solution $\left(u_{\mathrm{R}}(\xi), v_{\mathrm{R}}(\xi)\right)$ so that

$$
\left(u_{\mathrm{R}}, v_{\mathrm{R}}\right) \rightarrow(0,0) \text { as } \xi \rightarrow-\infty \quad \text { and } \quad\left(u_{\mathrm{R}}, v_{\mathrm{R}}\right) \rightarrow(1,0) \text { as } \xi \rightarrow+\infty .
$$

Linearise (4.1) about this wave:

$$
L_{\mathrm{R}} p=p^{\prime \prime}+c p^{\prime}+f^{\prime}\left(u_{\mathrm{R}}\right) p .
$$

Let $\sigma\left(L_{\mathrm{R}}\right)$ be the spectrum of $L_{\mathrm{R}}$ relative to $B=\mathrm{BC}(\mathbf{R}, \mathbf{R})$. Write $\left(L_{\mathrm{R}}-\lambda I\right) p=0$ as a system

$$
p^{\prime}=q, \quad q^{\prime}=-c q+\left(\lambda-f^{\prime}\left(u_{\mathrm{R}}\right)\right) p .
$$

This has an asymptotic system at $-\infty$,

$$
p^{\prime}=q, \quad q^{\prime}=-c q+\left(\lambda-f^{\prime}(0)\right) p,
$$

which I write as

$$
z^{\prime}=M_{0} z
$$

Set $S_{0}=\left\{\lambda: M_{0}(\lambda)\right.$ has an imaginary eigenvalue $\}$. 
There is an analogous picture at $+\infty$, where 0 is replaced by 1 throughout the above. Then $C \backslash S_{0} \cup S_{1}$ has a component $G_{R}$, containing the right half-plane, so that $\sigma\left(L_{\mathrm{R}}\right) \cap G_{\mathrm{R}} \subset \sigma_{n}\left(L_{\mathrm{R}}\right)$.

The function that plays the $D(\lambda)$ role can now be formulated. $M_{0}$ has eigenvalues and associated eigenvectors:

$$
\begin{array}{lll}
\mu^{+} & \left(\operatorname{Re} \mu^{+}>0\right) & X_{\mathbf{R}}^{+}, \\
\mu^{-} & \left(\operatorname{Re} \mu^{-}<0\right) & X_{\mathbf{R}}^{+}
\end{array}
$$

where

$$
\mu^{ \pm}=\left\{-c \pm\left(c^{2}+4\left(f^{\prime}(0)-\lambda\right)^{1 / 2}\right)\right\} / 2, \quad X_{R}^{ \pm}=\left(1, \mu^{ \pm}\right) .
$$

Write (4.4) as $z^{\prime}=M z$. Then for $\lambda \in G_{\mathrm{R}}$, there is a unique solution of $z^{\prime}=M z$ so that

$$
\zeta_{R}(\lambda, \xi)-X_{\mathrm{R}}^{+} e^{\mu^{+} \xi} \rightarrow 0 \text { as } \xi \rightarrow-\infty
$$

faster than $e^{\operatorname{Re} \mu^{+} \xi}$, and $\zeta_{R}(\lambda, \xi)$ is analytic in $\lambda$.

The adjoint equation is

$$
z^{* \prime}=N z^{*},
$$

where $N=-M^{*}$, and this has an asymptotic system

$$
z^{* \prime}=N_{1} z^{*},
$$

where $N_{1}=-M_{1}^{*} . N_{1}$ has eigenvalues and eigenvectors:

$$
\begin{array}{lll}
\nu^{+}=-\bar{\mu}^{+}, & \left(\operatorname{Re} \nu^{+}>0\right) & Y_{\mathrm{R}}^{+}=\left(1,\left(c-\nu^{+}\right)^{-1}\right), \\
\nu^{-}=-\bar{\mu}^{+}, & \left(\operatorname{Re} \nu^{-}<0\right) & Y_{\mathrm{R}}^{-}=\left(1,\left(c-\nu^{-}\right)^{-1}\right) .
\end{array}
$$

Also there is a unique solution of $(4.6), \eta_{R}(\lambda, \xi)$, so that

$$
\eta_{\mathrm{R}}(\lambda, \xi)-Y_{\mathrm{R}}^{-} e^{\nu^{-} \xi} \rightarrow 0 \text { as } \xi \rightarrow+\infty
$$

faster than $e^{\operatorname{Re} \nu^{-} \xi}$, and it is analytic in $\lambda . D_{R}(\lambda)$ is then defined as $\zeta_{R}(\lambda, \xi) \cdot \eta_{R}(\lambda, \xi)$. It has domain $G_{\mathrm{R}}$.

The stability of the travelling wave is well understood; see Fife and McLeod [13]. I shall translate the known facts into properties of $D_{R}(\lambda)$. This may seem to be backwards, but it is through $D_{R}(\lambda)$ that these known results will be used.

Facts about $D_{\mathrm{R}}(\lambda)$. (1) $D_{\mathrm{R}}(0)=0$.

(2) $D_{\mathrm{R}}(\lambda) \neq 0$ for $\lambda \in\{\lambda: \operatorname{Re} \lambda>d\}$, some $d<0$, except at 0 .

(3) $\left.(d / d \lambda) D_{R}(\lambda)\right|_{\lambda=0}>0$.

(1) follows from the standard feature of translation of waves. By a maximum principle argument, 0 is the eigenvalue of largest real part and there are only finitely many eigenvalues in $G_{\mathrm{R}}$; (2) therefore follows. I shall prove (3) directly in the following lemma.

LEMMA 4.1. $\left.(d / d \lambda) D_{\mathrm{R}}(\lambda)\right|_{\lambda-0}>0$. 
PRoof. To compute $(d / d \lambda) D_{\mathrm{R}}(\lambda)$ at $\lambda=0$, it suffices to consider $\lambda$ real. So $\zeta_{R}(\lambda, \xi) \in \mathbf{R}^{2}$ and $\eta_{R}(\lambda, \xi) \in \mathbf{R}^{2}$. Let $\zeta_{R}=\left(r_{R}, \theta_{R}\right)$ and $\eta_{R}=\left(r_{R}^{*}, \theta_{R}^{*}\right)$ in polar coordinates on the plane. Then

$$
D_{\mathrm{R}}(\lambda)=r_{\mathrm{R}} r_{\mathrm{R}}^{*} \cos \left(\theta_{\mathrm{R}}-\theta_{\mathrm{R}}^{*}\right) .
$$

Since $D_{\mathrm{R}}(0)=0$, one computes

$$
\frac{d}{d \lambda} D_{\mathrm{R}}(\lambda)=-r_{\mathrm{R}} r_{\mathrm{R}}^{*} \sin \left(\theta_{\mathrm{R}}-\theta_{\mathrm{R}}^{*}\right)\left\{\frac{\partial}{\partial \lambda} \theta_{\mathrm{R}}-\frac{\partial}{\partial \lambda} \theta_{\mathrm{R}}^{*}\right\}
$$

where the right-hand side can be evaluated at any $\xi$. Since $\theta_{R}-\theta_{R}^{*}=-\pi / 2$, at $\lambda=0$

$$
\frac{d}{d \lambda} D_{\mathrm{R}}(\lambda)=-r_{\mathrm{R}} r_{\mathrm{R}}^{*}\left\{\frac{\partial}{\partial \lambda} \theta_{\mathrm{R}}-\frac{\partial}{\partial \lambda} \theta_{\mathrm{R}}^{*}\right\}
$$

From (4.6)

$$
\theta_{\mathrm{R}}^{*} \rightarrow \arctan \left(\frac{1}{c-\nu_{-}}\right) \text {as } \xi \rightarrow+\infty
$$

and

$$
\frac{\partial}{\partial \lambda}\left\{\arctan \frac{1}{c-\nu_{-}}\right\}=\frac{\partial}{\partial \lambda}\left(\frac{\nu}{(c-\nu)^{2}+1}\right)<0
$$

since $\partial \nu_{-} / \partial \lambda<0$.

It follows that

$$
\lim _{\xi \rightarrow+\infty} \frac{\partial}{\partial \lambda} \theta_{R}^{*} \leqslant 0 .
$$

To check the term involving $\partial \theta_{\mathrm{R}} / \partial \lambda$, one computes

$$
\theta_{\mathrm{R}}^{\prime}=-c \sin \theta_{\mathrm{R}} \cos \theta_{\mathrm{R}}+\left(\lambda-f^{\prime}\left(u_{\mathrm{R}}\right)\right) \cos ^{2} \theta_{\mathrm{R}}-\sin ^{2} \theta_{\mathrm{R}}
$$

for $\theta_{R}$ as a function of $\xi$. From (4.7), if $\partial \theta_{R} / \partial \lambda=0$,

$$
\left\{\partial \theta_{\mathrm{R}} / \partial \lambda\right\}^{\prime}=\cos ^{2} \theta_{\mathrm{R}}>0 .
$$

By the same kind of argument as above,

$$
\lim _{\xi \rightarrow-\infty} \frac{\partial}{\partial \lambda} \theta_{R} \geqslant 0
$$

and so, from (4.7) and (4.8),

$$
\frac{\partial}{\partial \lambda} \theta_{R}>0 \text { for all } \xi, \quad \lim _{\xi \rightarrow+\infty} \inf \frac{\partial}{\partial \lambda} \theta_{R}>0 .
$$

It follows that $\partial \theta_{R} / \partial \lambda>0$, as desired.

If $\varepsilon=0$ and $w=0$ in (2.1), one obtains the system (4.2) coupled with the equation $w^{\prime}=0 . J_{\mathrm{F}}$ is a trajectory of $(2.1)$ in this invariant plane. The system restricted to this plane fits exactly into the form described in this section.

If $\varepsilon=0$ but $w=w^{*}$ (see $\S 2$ ), the equation for the back trajectory is obtained; it is

$$
u^{\prime}=v, \quad v^{\prime}=-c v-f(u)+w_{*}, \quad w^{\prime}=0 .
$$


If the third coordinate is dropped, one obtains a system that is analogous to the above reduced system. The nonlinearity $f(u)-w^{*}$ has the graph given in Figure 5 . The situation has merely been reversed; the right and left critical points have their roles interchanged. An analytic function is then defined for which the properties given for $D_{R}(\lambda)$ still hold.

5. Approximate location of eigenvalues. In this section I shall prove that any points in $\sigma(L) \cap G$ must lie close to eigenvalues of one of the reduced systems. It then follows that the only dangerous eigenvalues are close to zero.

The trajectories $J_{\mathrm{F}}$ and $J_{\mathrm{B}}$ described in $\S 2$ are, respectively, travelling waves for the PDEs

$$
u_{t}=u_{\xi \xi}+c^{*} u_{\xi}+f(u), \quad u_{t}=u_{\xi \xi}+c^{*} u_{\xi}+f(u)-w^{*} .
$$

As solutions to these equations call them $u_{F}(\xi)$ and $u_{B}(\xi)$, fixing some point at $\xi=0$, say $u_{F}(0)=a$ and $u_{B}(0)=0$. Let $L_{F}$ and $L_{B}$ be the linearised operators about these solutions. Let $\sigma_{\mathrm{F}}=\sigma\left(L_{\mathrm{F}}\right)$ and $\sigma_{\mathrm{B}}=\sigma\left(L_{\mathrm{B}}\right)$ relative to $B=\mathrm{BC}(\mathbf{R}, \mathbf{R})$.

Recall that $\tilde{G}=\{\lambda: \operatorname{Re} \lambda>b\}$. It is obvious that $b$ can be chosen so that $\tilde{G} \subset G_{\mathrm{F}} \cap G_{\mathrm{B}}$, where $G_{\mathrm{F}}=$ domain of $D_{\mathrm{F}}, G_{\mathrm{B}}=$ domain of $D_{\mathrm{B}}$, and $D_{\mathrm{F}}$ and $D_{\mathrm{B}}$ are the analytic functions for the front and back as given in $\S 4$.

Let $V=V_{\delta}=$ union of open balls of radius $\delta$ about each point in $\left(\sigma_{\mathrm{F}} \cup \sigma_{\mathrm{B}}\right) \cap \tilde{G}$. This section is devoted to proving the following theorem.

THEOREM 5.1. Given $\delta>0$ there exists $\varepsilon_{0}>0$ so that if $\varepsilon \in\left(0, \varepsilon_{0}\right], D(\lambda) \neq 0$ for $\lambda \in G \backslash V_{\delta}$.

Corollary 5.1. If $\lambda \in G \backslash V$ then $\lambda$ is not an eigenvalue of $L$.

The idea of the proof of Theorem 5.1 is to follow $\zeta(\lambda, \xi)$ until $\xi$ is very large and then evaluate $D(\lambda)$. If $\xi$ is large enough at the evaluation point, $\eta(\lambda, \xi)$ is essentially determined there.

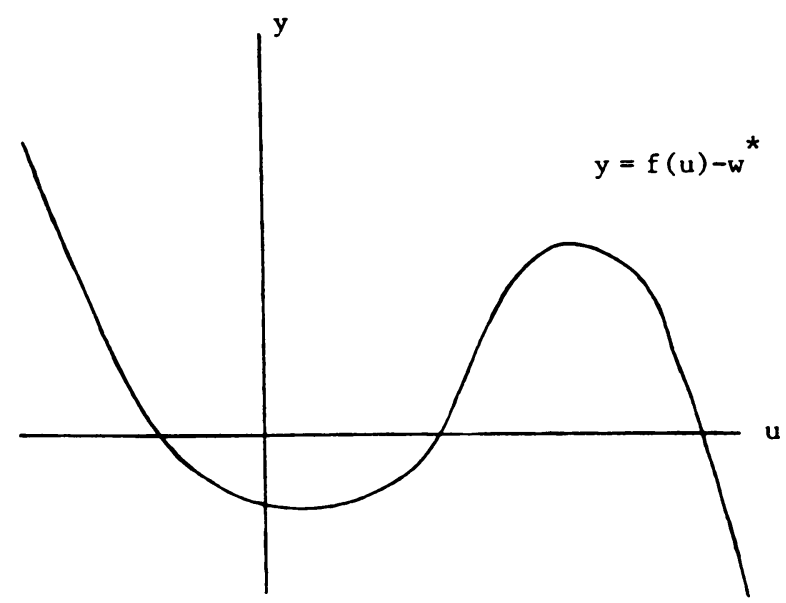

Figure 5 
Following $\zeta(\lambda, \xi)$ as $\xi$ varies can be thought of as following it "around" the travelling wave. $\zeta(\lambda, \xi)$ satisfies (3.2), its dependence on $\xi$ is through $u(\xi)$, the first component of the travelling wave $U(\xi)$. If a copy of $\mathbf{C}^{3}$ is attached to each point of the orbit $\left(S_{\varepsilon}\right), \zeta(\lambda, \xi)$ lies in that copy if $U(\xi)$ is the underlying point.

To make this more precise, couple the travelling wave system (2.1) with the eigenvalue system (3.2),

$$
\begin{gathered}
u^{\prime}=v, \quad v^{\prime}=-c v-f(u)+w, \quad w^{\prime}-(\varepsilon / c)(u-\gamma w), \quad p^{\prime}=q, \\
q^{\prime}=-c q+\left(\lambda-f^{\prime}(u)\right) p+r, \quad r^{\prime}=-(\varepsilon / c) p+[(\lambda+\varepsilon \gamma) / c] r,
\end{gathered}
$$

where $U=(u, v, w) \in \mathbf{R}^{3}$ and $z=(p, q, r) \in \mathbf{C}^{3}$. The natural setting for (5.1) is the complexified tangent bundle to $\mathbf{R}^{3}$, denoted $T_{c} \mathbf{R}^{3}$. This is isomorphic to $\mathbf{R}^{3} \times \mathbf{C}^{3}$. (5.1) induces a flow on $T_{\mathrm{c}} \mathbf{R}^{3}$ that depends continuously on $(\lambda, c, \varepsilon) \in \mathbf{C} \times \mathbf{R} \times \mathbf{R}$.

The travelling wave for $\varepsilon \neq 0$ is denoted $S_{\varepsilon} \subset \mathbf{R}^{3}$, with $c=\bar{c}(\varepsilon)$. If the flow above is restricted to $S_{\varepsilon}$, we obtain a flow on $S_{\varepsilon} \times \mathbf{C}^{3}$, the component on $\mathbf{C}^{3}$ coming from (3.2). This flow depends on $\lambda \in \mathbf{C}$ and is defined for $\varepsilon \in\left[0, \varepsilon_{0}\right]$.

$\zeta(\lambda, \xi)$ will be followed around $S_{\varepsilon} \times \mathbf{C}^{3}$ as a trajectory for this flow, i.e. $(u(\xi), \zeta(\lambda, \xi))$ will be followed as $U(\xi)$ goes around $S_{\xi}$.

Not all of the information in $\zeta(\lambda, \xi)$ will be necessary to deduce $D(\lambda) \neq 0$. In fact, only its "direction" is important; the appropriate context is the projectivised space.

Since (5.1) is linear in $z \in \mathbf{C}^{3}$, the flow can be projectivised in the second component. Using coordinates $(U, z) \in T_{\mathrm{c}} \mathbf{R}^{3}, P T_{\mathrm{c}} \mathbf{R}^{3}=\mathbf{R}^{3} \times \mathbf{C}^{3} \backslash\{0\} / \sim$, where $\left(U_{1}, z_{1}\right) \sim\left(U_{2}, z_{2}\right)$ if $U_{1}=U_{2}$ and there exists an $\alpha \in \mathbf{C}$ so that $z_{1}=\alpha z_{2}$.

Clearly $P T_{\mathrm{c}} \mathbf{R}^{3} \simeq \mathbf{R}^{3} \times \mathrm{CP}^{2}$, where $\mathrm{CP}^{2}$ is two-dimensional complex projective space. Let $\pi: \mathrm{C}^{3} \rightarrow \mathrm{CP}^{2}$ be the natural map; $\pi(z)$ is the equivalence class determined by $z$, which is $\operatorname{span}_{\mathbf{C}}\{z\} \backslash\{0\}$. I shall use the notation $\hat{z}=\pi(z)$. Extend $\pi$ to $P T_{\mathrm{c}} \mathbf{R}^{3}$ :

$$
\pi: T_{\mathrm{c}} \mathbf{R}^{3} \rightarrow P T_{\mathrm{c}} \mathbf{R}^{3}, \quad(U, z) \rightarrow(U, \hat{z}) .
$$

Since (5.1) is linear, it induces a flow on $P T_{\mathrm{c}} \mathbf{R}^{3}$. If $F(\xi)$ is the time- $\xi$ map of (5.1), then $\hat{F}(\xi)$, the time- $\xi$ map of the projectivized flow is the unique map for which the diagram

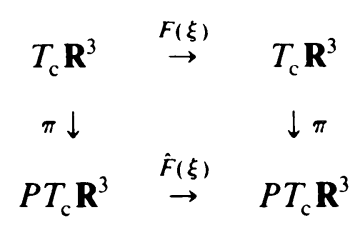

commutes. All continuous dependence on parameters is inherited by $\hat{F}(\xi)$.

$S_{\varepsilon} \times \mathbf{C P}^{2}$ is an invariant subspace of $P T_{\mathrm{c}} \mathbf{R}^{3}$ for fixed $\varepsilon$. There is therefore a (global, since the space is compact) flow on $S_{\varepsilon} \times \mathrm{CP}^{2}$ depending on $\lambda \in \mathbf{C}$. Using Theorem 2.1 the flow on $\cup S_{\varepsilon} \times \mathbf{C P}^{2}$, where the union is over $\varepsilon \in\left[0, \varepsilon_{0}\right]$, can be considered to lie on $S^{1} \times\left[0, \varepsilon_{0}\right] \times \mathbf{C P}^{2}$.

Local coordinates can be put on $\mathbf{C P}^{2}$ in the following way. Let $z=(p, q, r) \in \mathbf{C}^{3}$ and $\pi(z) \in \mathrm{CP}^{2}$. If $p \neq 0$, then $\pi(z)$ is given by the coordinates $(q / p, r / p)$. This is 
obviously independent of which point in $\pi^{-1}(\pi(z))$ is used. Each of the other components can be used to get other local coordinate systems, but I shall aiways use the above.

For each $\hat{z} \in \mathbf{C P}^{2}$, so that $\hat{z}=(q / p, r / p)$, there is a distinguished vector in $\mathbf{C}^{3}$, call it $\tilde{z}=(1, q / p, r / p)$, so that $\pi(\tilde{z})=\pi(z)$. In other words $\tilde{z}$ is a normalised version of $z$.

The adjoint system (3.10) can be dealt with similarly. The natural phase space here is the projectivised, complexified cotangent bundle $P T_{c}^{*} \mathbf{R}^{3}$ ! I shall not use this at all, however.

Now let $\zeta(\lambda, \xi)$ and $\eta(\lambda, \xi)$ have their usual meanings. Suppose at a certain value of $\xi, \zeta(\lambda, \xi)$ and $\eta(\lambda, \xi)$ are well defined. It is clear that if $\tilde{\zeta}(\lambda, \xi) \cdot \tilde{\eta}(\lambda, \xi) \neq 0$ then $\zeta(\lambda, \xi) \cdot \eta(\lambda, \xi) \neq 0$. This proves the following lemma.

LEMMA 5.1. If $\lambda \in \tilde{G}$ and there exists $a \xi \in \mathbf{R}$, so that $\tilde{\zeta}(\lambda, \xi) \cdot \tilde{\eta}(\lambda, \xi) \neq 0$, then $\tilde{D}(\lambda) \neq 0$.

The asymptotic systems are constant coefficient linear systems on $\mathbf{C}^{3}$. By projectivising such an autonomous linear flow, one obtains a flow on $\mathrm{CP}^{2}$. The following special considerations apply.

Let $A: \mathbf{C}^{3} \rightarrow \mathrm{C}^{3}$ be linear. Then $A$ induces a vector field on $\mathrm{CP}^{2}$ as follows:

$$
\begin{array}{ccc}
\mathrm{C}^{3} & \stackrel{(\text { id. } A)}{\rightarrow} & T \mathrm{C}^{3} \\
\pi \downarrow & & \downarrow D \pi \\
\mathrm{CP}^{2} & \stackrel{g}{\rightarrow} & T \mathrm{CP}^{2}
\end{array}
$$

I shall call it $g$.

Let $C_{\alpha}$ be a one-dimensional eigenspace for $A$ associated with an eigenvalue $\alpha$. $\pi\left(C_{\alpha}\right)$ is then a critical point for the flow on $\mathrm{CP}^{2}$. If one linearises $g$ at $\pi\left(C_{\alpha}\right)$, $D g\left(\pi\left(C_{\alpha}\right)\right)$ can be considered as a linear map on $C^{2}$. I shall prove the following lemma.

LEMma 5.2. If $A$ has eigenvalues $\alpha, \alpha_{1}, \alpha_{2}$, and $\alpha$ is simple, then $D g\left(\pi\left(C_{\alpha}\right)\right)$ : $\mathrm{C}^{2} \rightarrow \mathrm{C}^{2}$ has eigenvalues $\alpha_{1}-\alpha$ and $\alpha_{2}-\alpha$.

Proof. Suppose $\alpha_{1} \neq \alpha_{2}$. Then choose a basis for $C^{3}$ in which $A$ becomes

$$
\left(\begin{array}{ccc}
\alpha & 0 & 0 \\
0 & \alpha_{1} & 0 \\
0 & 0 & \alpha_{2}
\end{array}\right)
$$

Let $(p, q, r)$ be the coordinates in this basis. Then, near $\pi\left(C_{\alpha}\right),(q / p, r / p)$ are local coordinates on $\mathrm{CP}^{2}$. Consider $D \pi: T \mathrm{C}^{3} \rightarrow T \mathrm{CP}^{2}$; let $\left((p, q, r),\left(z_{1}, z_{2}, z_{3}\right)\right)$ be a generic point in $T \mathrm{C}^{3}$. Then

$$
D \pi\left((p, q, r),\left(z_{1}, z_{2}, z_{3}\right)\right)=\left(\left(\frac{q}{p}, \frac{r}{p}\right),\left(\frac{z_{2}}{p}-\left(\frac{q}{p}\right) \frac{z_{1}}{p}, \frac{z_{3}}{p}-\left(\frac{r}{p}\right) \frac{z_{1}}{p}\right)\right)
$$


from the quotient rule. Chasing a point around (5.2):

$$
\begin{array}{ccc}
(p, q, r) & \rightarrow & \left((p, q, r),\left(\alpha p, \alpha_{1} q, \alpha_{2} r\right)\right) \\
\downarrow & \downarrow & \downarrow \\
\left(\frac{q}{p}, \frac{r}{p}\right) & \rightarrow & \left(\left(\frac{q}{p}, \frac{r}{p}\right),\left(\left(\alpha_{1}-\alpha\right) \frac{q}{p},\left(\alpha_{2}-\alpha\right) \frac{r}{p}\right)\right)
\end{array}
$$

In these coordinates the second component is linear and is therefore $\operatorname{Dg}\left(\pi\left(C_{\alpha}\right)\right)$. As a matrix it has the form

$$
\left(\begin{array}{cc}
\alpha_{1}-\alpha & 0 \\
0 & \alpha_{2}-\alpha
\end{array}\right)
$$

and the lemma holds for this case. If $\alpha_{1}=\alpha_{2}$ and the geometric multiplicity is only one, $\operatorname{Dg}\left(\pi\left(C_{\alpha}\right)\right)$ would be

$$
\left(\begin{array}{cc}
\alpha_{1}-\alpha & 0 \\
1 & \alpha_{2}-\alpha
\end{array}\right)
$$

and the result still holds.

As an application of this lemma, consider the linear equation with constant coefficients $z^{\prime}=A z$. If $\alpha$ is the eigenvalue of $A$ of largest real part, then $C_{\alpha} \in \mathbf{C P}^{2}$ is an attracting critical point. Similarly, if $\alpha$ were of smallest real part, $C_{\alpha}$ would be a repelling critical point. Paraphrasing this, one can say that unstable subspaces become stable critical points and stable subspaces become unstable critical points.

To prove Theorem 5.1, I shall divide $G \backslash V$ into two sets:

$$
\begin{aligned}
& G_{1}=\{\lambda: \lambda \in G \backslash V \text { and }|\lambda|>k\} \quad \text { for some fixed } k>0 . \\
& G_{2}=\{G \backslash V\} \backslash G_{1} .
\end{aligned}
$$

Evans [9] proves an asymptotic estimate for $|\lambda| \rightarrow+\infty$ that shows if $\lambda \in G_{1}$ for some $k>0$, then $\lambda$ is not an eigenvalue, i.e., $D(\lambda) \neq 0$.

The main task then is to prove that for $\lambda \in G_{2}, D(\lambda) \neq 0$. This will be proved for any $k . \hat{\zeta}(\lambda, \xi)$ will be followed around $S_{\varepsilon}$, and then at large $\xi, \hat{\zeta}(\lambda, \xi)$ will be used to determine $\tilde{\zeta}(\lambda, \xi) . \tilde{\zeta} \cdot \tilde{\eta}$ will then be proved to be nonzero so, by Lemma $5.1, \lambda$ could not be an eigenvalue.

I shall actually restrict $\lambda$ to a larger set than $G_{2}$. Let

$$
\Omega=\{\lambda \in \operatorname{cl}(\tilde{G}): \lambda \notin V \text { and }|\lambda| \leqslant k\} .
$$

Then $G_{2} \subset \Omega$ and $\Omega$ is compact. It follows from Lemma 3.3 that $\xi(\lambda, \xi), \eta(\lambda, \xi)$ and $D(\lambda)$ are all defined in $\Omega$ and analytic in $\operatorname{int}(\Omega)$.

There are various flows I shall want to consider, depending on how many parameters are fixed. As stated earlier, the full equations (5.1) induce a flow on $\bigcup_{0 \leqslant \varepsilon \leqslant \varepsilon_{0}} S_{\varepsilon} \times \mathbf{C P}^{2}$, where $\varepsilon_{0}$ satisfies all the requirements collected to date. Using $h$ : $S^{1} \times\left[0, \varepsilon_{0}\right] \rightarrow \cup S_{\varepsilon}$, from Theorem 2.1, there is a flow on $S^{1} \times\left[0, \varepsilon_{0}\right] \times \mathbf{C P}^{2}$.

With the parameter $\lambda$ set by the flow, there is a flow on $S^{1} \times\left[0, \varepsilon_{0}\right] \times \mathbf{C P}^{2} \times \Omega$. Call this flow $H(t)$. If $\lambda$ is fixed, let $H^{\lambda}(t)$ be the flow on $S^{1} \times\left[0, \varepsilon_{0}\right] \times \mathrm{CP}^{2} . H_{\varepsilon}(t)$ and $H_{\varepsilon}^{\lambda}(t)$ then have the obvious meaning. 
Control on $\hat{\zeta}(\lambda, \xi)$ will be afforded by proving certain properties for the flow $H_{0}(t)$ on $S^{1} \times \mathrm{CP}^{2}$ and then perturbing this information to $H_{\varepsilon}(t)$.

Recall the construction of a tubular neighborhood about the singular orbit $S_{0}$ given in the proof of Theorem 2.1. The corner boxes are $B_{0}, B_{1}, B_{2}, B_{3}$. Let the following points $b_{i}, i=0,1,2,3$, be the indicated crossing points of $S_{0}$ with the respective boxes:

$$
\begin{array}{ll}
b_{0} \in S_{0} \cap \partial B_{0} \cap\left\{u=\gamma_{1}\right\}, & b_{1} \in S_{0} \cap \partial B_{1} \cap\left\{u=u_{1}-\gamma_{1}\right\}, \\
b_{2} \in S_{0} \cap \partial B_{2} \cap\left\{u=u_{2}-\gamma_{1}\right\}, & b_{3} \in S_{0} \cap \partial B_{3} \cap\left\{u=u_{3}+\gamma_{1}\right\} .
\end{array}
$$

All notation is defined in §2; see the proof of Theorem 2.1. Recall that $h_{0}=h \mid S^{1} \times$ $\{0\}$. Let $\theta_{i}$ be given by the condition

$$
h_{0}\left(\theta_{i}\right)=b_{i}, \quad i=0,1,2,3 .
$$

Recall further from $\S 3$ that $U_{0}, U_{1}, U_{2}$ and $U_{3}$ denote the four corners of $S_{0}$; see Figure 2. Let $\bar{\theta}_{i}$ be determined by the conditions

$$
h_{0}\left(\theta_{i}\right)=U_{i}, \quad i=0,1,2,3 \text {. }
$$

The first property of the $H_{0}$ flow is that it possesses a certain attractor that sits over the right-hand slow manifold in the construction of $S_{0}$.

The attractor will be a set of points of the form $(\theta, \hat{X}(\theta, \lambda), \lambda) \in S^{1} \times \mathbf{C P}^{2} \times \Omega$, where $\theta \in\left[\bar{\theta}_{1}, \theta_{2}\right]$. It will then be

$$
K=\bigcup_{\bar{\theta}_{1} \leqslant \theta \leqslant \theta_{2}} \bigcup_{\lambda \in \Omega}(\theta, \hat{X}(\theta, \lambda), \lambda)
$$

In order to describe $\hat{X}(\theta, \lambda)$, let $u_{\theta}=u$-component of $h_{0}(\theta) \in S_{0} \subset \mathbf{R}^{3} .\{\theta\} \times$ $\mathrm{CP}^{2}$ is an invariant subset of $S^{1} \times \mathrm{CP}^{2}$ under $H_{0}(t)$ if $\bar{\theta}_{1} \leqslant \theta \leqslant \bar{\theta}_{2}$. The flow on $\mathrm{CP}^{2}$ is the projectivised version of

$$
p^{\prime}=q, \quad q^{\prime}=-c q+\left(\lambda-f^{\prime}\left(u_{\theta}\right)\right)_{p}, \quad r^{\prime}=0
$$

$b$ can be chosen, to set $\tilde{G}$, so that (5.4) has a unique simple eigenvalue of largest real part for each $(\theta, \lambda)$; call it $\alpha^{+}(\theta, \lambda)$. Call some associated eigenvector $X^{+}(\theta, \lambda)$. $\hat{X}(\theta, \lambda)$ is then set as $\hat{X}^{+}(\theta, \lambda)$.

The set

$$
K_{1}=\bigcup_{\bar{\theta}_{1} \leqslant \theta \leqslant \bar{\theta}_{2}} \bigcup_{\lambda \in \Omega}(\theta, \hat{X}(\theta, \lambda), \lambda)
$$

will form part of the attractor. It needs to be extended to $\theta_{2}$, i.e., away from the corner to the edge of $B_{2}$.

Let $\theta \in\left[\bar{\theta}_{2}, \theta_{2}\right]$. Then $h_{0}(\theta) \in J_{\mathrm{B}} . J_{\mathrm{B}}$ is parametrised by $\xi \in \mathbf{R}$ and given by $\left(u_{\mathrm{B}}(\xi), u_{\mathrm{B}}^{\prime}(\xi), w^{*}\right)$. From $\S 4$ there exists a uniquely determined, up to normalisation, solution for the linearised eigenvalue equations over the back; call this $\zeta_{B}(\lambda, \xi)$. Now set $\hat{X}(\theta, \lambda)=\hat{\zeta}_{B}(\lambda, \xi)$, where $\theta$ and $\xi$ are related by the condition $\theta_{B}(\xi)=\theta$.

Now $\hat{X}(\theta, \lambda)$ is defined for all $\theta \in\left[\bar{\theta}_{1}, \theta_{2}\right]$. The attractor is then the set $K$ given by (5.3). I need to show that $K$ is an attractor in some suitable sense.

LEMMA 5.3. $K$ is an attractor for the flow $H_{0}(t)$ relative to the set

$$
\left[0, \theta_{2}\right] \times \mathbf{C P}^{2} \times \Omega=F .
$$

In other words, there is a neighborhood $Q$ of $K$ in $F$ so that $\omega(Q) \cap F=K$. 
REMARK. $\theta_{2}$ depends on the size of the tubular neighborhood, i.e. $\kappa$. Lemma 5.3 holds for all sufficiently small $\kappa$.

Proof. First consider the set $K_{1}$. Since $X^{+}(\theta, \lambda)$ is an eigenvector for the eigenvalue of largest real part, it follows from Lemma 5.2 that $\left(\theta, \hat{X}^{+}(\theta, \lambda)\right)$ is an attractor in $\{\boldsymbol{\theta}\} \times \mathbf{C P}^{2}$ for each fixed $\lambda$.

To show that $K_{1}$ is an attractor it suffices to show that the rate of convergence to $(\theta, \hat{X}(\theta, \lambda))$ is bounded away from zero uniformly in $\theta \in\left[\bar{\theta}_{1}, \bar{\theta}_{2}\right]$ and $\lambda \in \Omega$. $\alpha^{+}(\theta, \lambda)$ depends continuously on $\theta$ and $\lambda$; moreover, the rate of convergence to the attracting point $\left(\theta, \hat{X}^{+}(\theta, \lambda)\right)$ in $\{\theta\} \times \mathbf{C P}^{2}$ is determined by the quantity

$$
\operatorname{Re}\left(\alpha^{+}(\theta, \lambda)-\alpha^{0}(\theta, \lambda)\right)
$$

where $\alpha^{0}(\theta, \lambda)$ is the eigenvalue of next largest real part. In the proof of Lemma 3.3, it is shown that (5.6) is bounded away from zero and positive if $\lambda \in \tilde{G}$. (5.4) is the same as (3.5) except that 0 is replaced by $u_{\theta}$. But there is an $a<0$ so that $f^{\prime}\left(u_{\theta}\right)<a<0$ for all $\theta \in\left[\bar{\theta}_{1}, \bar{\theta}_{2}\right]$, so it is clear that this also holds here. By compactness of $\left[\bar{\theta}_{1}, \bar{\theta}_{2}\right] \times \Omega,(5.6)$ can be uniformly bounded away from zero.

It follows that $K_{1}$ is an attractor relative to the set

$$
\left[\bar{\theta}_{1}, \bar{\theta}_{2}\right] \times \mathrm{CP}^{2} \times \Omega \text {. }
$$

I claim that it is, in fact, an attractor in the set

$$
\left[0, \bar{\theta}_{2}\right] \times \mathrm{CP}^{2} \times \Omega \text {. }
$$

Relative to $\left[0, \bar{\theta}_{1}\right] \times \mathbf{C P}^{2} \times \Omega$, the invariant set $\overline{\boldsymbol{\theta}}_{1} \times \mathbf{C P}^{2} \times \Omega$ is itself an attractor. This is trivial because the underlying flow on $\left(0, \bar{\theta}_{1}\right)$ is just the front solution (see Figure 6) and increases to $\bar{\theta}_{1}$. It can then also be said that (5.7) is an attractor relative to the set of (5.8). $K_{1}$ is therefore an attractor within an attracting invariant set and so is an attractor in (5.8).

The full attractor $K$ is $K_{1}$ with a piece put on the tail. It suffices to show that the tail

$$
K_{2}=\bigcup_{\bar{\theta}_{2} \leqslant \lambda \leqslant \theta_{2}} \bigcup_{\lambda \in \Omega}(\theta, \hat{X}(\theta, \lambda), \lambda)
$$

is an attractor relative to the set

$$
\left[\bar{\theta}_{2}, \theta_{2}\right] \times \mathrm{CP}^{2} \times \Omega .
$$

To this end, consider the flow induced on $\mathbf{R}^{3} \times \mathbf{C P}^{2}$ from (5.1). Appending $\lambda$, there is a flow on $\mathbf{R}^{3} \times \mathbf{C P}^{2} \times \Omega$. The point $k_{2}(\lambda)=\left(U_{2}, \hat{X}^{+}\left(\bar{\theta}_{2}, \lambda\right), \lambda\right)$ is a critical point for each fixed $\lambda$. The linearisation in $\mathbf{R}^{3} \times \mathbf{C P}^{2} \times \Omega$ has one eigenvalue of positive real part, three of zero real part and the rest of negative real part. $\lambda$ and $w$ determine the ones of zero real part.

The point $k_{2}$, therefore, has a (real) four-dimensional center-unstable manifold $W^{\text {cu }}\left(k_{2}\right)$, which is attracting relative to a compact neighborhood of $k_{2}(\lambda)$, say $V(\lambda)$. Set $V=\bigcup_{\lambda \in \Omega} V(\lambda)$ and $W=\bigcup_{\lambda \in \Omega}\left(W^{\text {cu }}\left(k_{2}(\lambda)\right) \cap V(\lambda)\right)$. Then $W \subset \mathbf{R}^{3} \times \mathbf{C P}^{2} \times \Omega$ and is attracting relative to $V$. Let $K_{3}=W \cap\left(J_{\mathbf{B}} \times \mathbf{C P}^{2} \times \Omega\right)$. Fix $\lambda_{0} \in \Omega$ and define $K_{3}\left(\lambda_{0}\right)=K_{3} \cap\left\{\lambda=\lambda_{0}\right\}$. Notice that the critical point $k_{2}(\lambda) \in K_{3}(\lambda)$. It is easy to check that $K_{3}(\lambda)$ contains none of the center directions in $W^{\text {cu }}\left(k_{2}\right)$. 


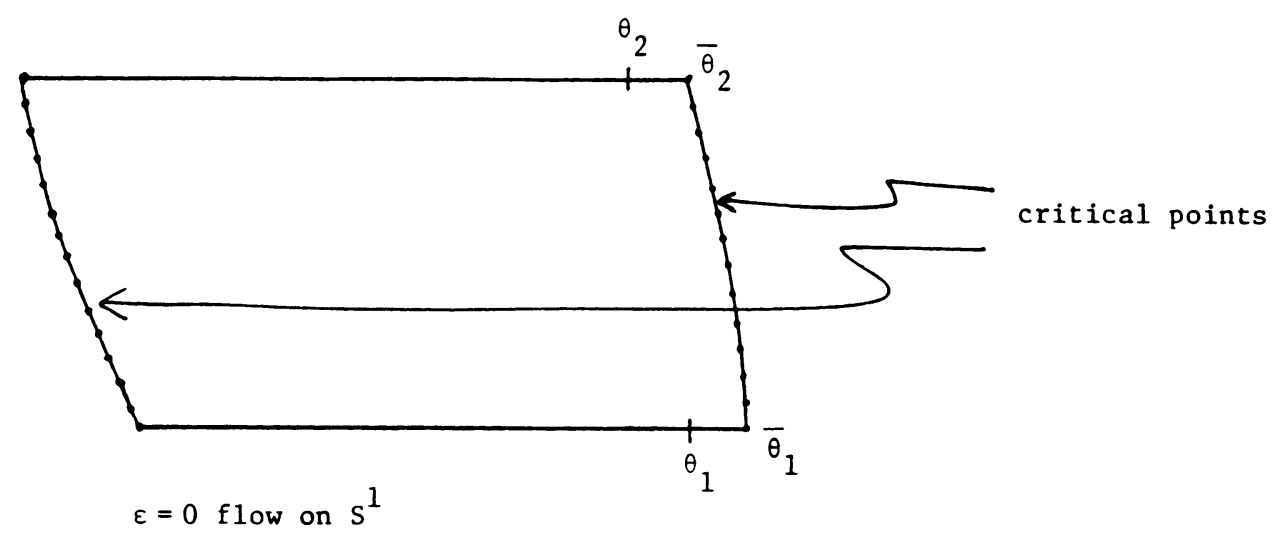

Figure 6

Therefore $K_{3}(\lambda)$ is one-dimensional and must lie in $W^{\mathrm{u}}\left(k_{2}\right)$. It is therefore a curve of the form $\left(U_{\mathrm{B}}(\xi), \hat{X}(\lambda, \xi)\right)$, where $U_{\mathrm{B}}(\xi)$ is the solution corresponding to $J_{\mathrm{B}} \subset \mathbf{R}^{3}$, the back. The above is a trajectory in the ( $\lambda$ fixed) flow on $S_{0} \times \mathrm{CP}^{2}$. Furthermore, it must satisfy, as $\xi \rightarrow-\infty,\left(U_{\mathrm{B}}(\xi), \hat{X}(\lambda, \xi)\right) \rightarrow\left(U_{2}, \hat{X}^{+}\left(\bar{\theta}_{2}, \lambda\right)\right)$.

But there is a unique curve that does this, namely $\left(U_{\mathrm{B}}(\xi), \hat{\zeta}_{\mathrm{B}}(\lambda, \xi)\right)$.

Now extend $h_{0}: S^{1} \rightarrow S_{0}$ to $\tilde{h}_{0}: S^{1} \times \mathrm{CP}^{2} \times \Omega \rightarrow S_{0} \times \mathrm{CP}^{2} \times \Omega$ by the identity.

Define $K_{2}$ by the condition $h_{0}\left(K_{2}\right)=K_{3}$. $K_{2}$ is of the form

$$
\bigcup_{\bar{\theta}_{2} \leqslant \theta \leqslant \eta} \bigcup_{\lambda \in \Omega}(\theta, \hat{X}(\theta, \lambda), \lambda)
$$

for some $\eta>\bar{\theta}_{2}$. Choose $K$ and, therefore, set $\theta_{2}$ so that $\theta_{2}<\eta$. Finally, reset $V$ and, hence, $K_{2}$ so that $\eta=\theta_{2}$.

Since $K_{3}$ is an attractor in $V \cap\left(J_{\mathrm{B}} \times \mathrm{CP}^{2} \times \Omega\right)$, the same is true of $K_{2}$ in $\left[\bar{\theta}_{2}, \theta_{2}\right] \times \mathrm{CP}^{2} \times \Omega$. Also, the only exit set in the boundary of the neighborhood lies in $\left\{\theta_{2}\right\} \times \mathrm{CP}^{2} \times \Omega$. It follows that, if this neighborhood is called $Q_{2}$,

$$
\omega\left(Q_{2}\right) \cap\left[\bar{\theta}_{2}, \theta_{2}\right] \times \mathrm{CP}^{2} \times \Omega=K_{2} .
$$

Now choose a neighborhood $Q_{1}$ of $K_{1}$ in $\left[0, \bar{\theta}_{2}\right] \times \mathbf{C P}^{2} \times \Omega$. Since $K_{1} \cap\left\{\bar{\theta}_{2}\right\} \times$ $\mathbf{C P}^{2} \times \Omega=K_{2} \cap\left\{\bar{\theta}_{2}\right\} \times \mathbf{C P}^{2} \times \Omega$, one can choose a $Q_{1}$ so that $Q_{1} \cap\left\{\theta=\bar{\theta}_{2}\right\}=$ $Q_{2} \cap\left\{\theta=\bar{\theta}_{2}\right\}$. Let $Q=Q_{1} \cup Q_{2}$. It is then not hard to see that $Q$ is an attracting neighborhood of $K$ relative to $\left[0, \theta_{2}\right] \times \mathrm{CP}^{2} \times \Omega$.

The proof of Theorem 5.1 also needs an attractor that sits over the left-hand manifold. This would be a set of the form

$$
K_{\mathrm{L}}=\bigcup_{\bar{\theta}_{3} \leqslant \theta \leqslant 2 \pi} \bigcup_{\lambda \in \Omega}(\theta, \hat{X}(\theta, \lambda), \lambda),
$$

where $\hat{X}(\theta, \lambda)$ is again the projectivised unstable eigenvector of the appropriate system.

The fact that this is an attractor is significantly easier to prove than for $K$ because it lacks the tail of $K$. I shall only give the proof for $K$.

The following lemma about the behavior of the reduced system on the projectivised level will play a central role. 
LEMMA 5.4. Let $C \subset \Omega$ be a compact set so that $C \subset \operatorname{dom}\left(D_{\mathrm{R}}\right)=G_{\mathrm{R}}$ and $D_{\mathrm{R}}(\lambda) \neq 0$ for all $\lambda \in C$. Then

$$
\lim _{\xi \rightarrow+\infty} \hat{\zeta}_{R}(\lambda, \xi)=\hat{X}_{1}^{+}(\lambda)
$$

uniformly for $\lambda \in C . X_{1}^{+}(\lambda)$ is the eigenvector whose eigenvalue has positive real part for the system $z^{\prime}=M_{1} z$ (see $\left.\$ 4\right)$.

REMARK. The projectivising here is restricted to $\mathbf{C}^{2}$. So if $X \in \mathbf{C}^{2}, \hat{X} \in \mathbf{C P}^{1}$.

PROoF. $\zeta_{R}(\lambda, \xi)$ satisfies (4.4), which, when coupled with the travelling wave equations (4.2), gives a system on $\mathbf{R}^{2} \times \mathbf{C}^{3}$. When projectivised this leaves a system on $\mathbf{R}^{2} \times \mathbf{C P}$. Let $J_{\mathrm{R}} \subset \mathbf{R}^{2}$ be the closure of the wave trajectory; then $J_{\mathrm{R}} \times \mathbf{C P}^{1}$ is invariant.

The asymptotic system lies at $(1,0) \times \mathrm{CP}^{1}$ and is given by (4.5) with 0 replaced by 1. $M_{1}$ has two eigenvalues $\mu_{1}^{ \pm}$and associated eigenvectors $X_{1}^{ \pm}$. The projectivised flow on $\mathbf{C P}^{1}$, therefore, has two critical points $\hat{X}_{1}^{ \pm} \cdot \hat{\zeta}_{R}(\lambda, \xi)$ must tend to one of these as $\xi \rightarrow+\infty$. The set $\omega\left(u_{R}(\bar{\xi}), \hat{\zeta}_{R}(\lambda, \bar{\xi})\right)$, some $\bar{\xi}$, is an invariant subset of $\{(1,0)\} \times \mathbf{C P}^{1}$ and is therefore one of the critical points.

If $\hat{\zeta}_{R}(\lambda, \xi) \rightarrow \hat{X}_{1}^{-}(\lambda), \lambda$ would be an eigenvalue. By assumption it is not. Therefore $\hat{\zeta}_{R}(\lambda, \xi) \rightarrow \hat{X}_{1}^{+}(\lambda)$.

That the convergence is uniform follows from the fact that the rate of convergence to $\hat{X}_{1}^{+}(\lambda)$ in $(1,0) \times \mathrm{CP}^{2}$ is determined by $\operatorname{Re}\left(\mu^{-}(\lambda)-\mu^{+}(\lambda)\right)$, which can be seen to be bounded uniformly from zero, as it is continuous.

This is all the machinery I need to establish the central estimates in the proof of Theorem 5.1.

Let $\theta_{\varepsilon}(\xi)$ be the parameterisation induced on $S_{\varepsilon}$ by the travelling wave equations. Define $T_{i}=T_{i}(\varepsilon)$ by

$$
\theta_{\varepsilon}\left(T_{i}\right)=\theta_{i}, \quad i=0,1,2,3 .
$$

I shall evaluate $\hat{\xi}\left(\lambda, T_{i}\right)$ for $i=0,1,2,3$ and $\hat{\zeta}\left(\lambda, T_{4}\right)$ where $T_{4}$ is very large.

Now let $j: \mathbf{C}^{2} \rightarrow \mathbf{C}^{3}$ be the inclusion map $j(p, q)=(p, q, 0)$. Let $\zeta_{\mathrm{R}}(\lambda, \xi)$ be the eigenvalue solution for (4.4), i.e., the reduced system. Set $\zeta_{F}(\lambda, \xi)=j\left(\zeta_{R}(\lambda, \xi)\right)$. Notice that this coincides with $\zeta(\lambda, \xi)$ provided in $\S 3$ for $\varepsilon=0$.

Formulate the reduced system appropriate for the back; see comment at end of §4. Let $\zeta_{R}^{\prime}(\lambda, \xi)$ be the eigenvalue solution. Set $\zeta_{B}(\lambda, \xi)=j\left(\zeta_{R}^{\prime}(\lambda, \xi)\right)$.

Let $\theta_{F}(\xi)$ and $\theta_{B}(\xi)$ be the parametrisations induced on $S^{1} \times\{0\}$, from $S_{0}$, which correspond, respectively, to the front and the back. They should each be normalised in some fashion.

Set $T_{0}^{\mathrm{F}}, T_{1}^{\mathrm{F}}, T_{2}^{\mathrm{B}}$ and $T_{3}^{\mathrm{B}}$ by

$$
\boldsymbol{\theta}_{\mathrm{F}}\left(T_{0}^{\mathrm{F}}\right)=\boldsymbol{\theta}_{0}, \quad \boldsymbol{\theta}_{\mathrm{F}}\left(T_{1}^{\mathrm{F}}\right)=\boldsymbol{\theta}_{1}, \quad \boldsymbol{\theta}_{\mathrm{B}}\left(T_{2}^{\mathrm{B}}\right)=\boldsymbol{\theta}_{2}, \quad \boldsymbol{\theta}_{\mathrm{B}}\left(T_{3}^{\mathrm{B}}\right)=\boldsymbol{\theta}_{3} .
$$

In other words, these are the times at which the singular orbit $S_{0}$ hits the box edges.

I shall prove the theorem by five estimates of the following form:

(1) $\left|\tilde{\zeta}\left(\lambda, T_{0}\right)-\tilde{\zeta}_{\mathrm{F}}\left(\lambda, T_{0}^{\mathrm{F}}\right)\right|<\delta_{0}$;

(2) $\left|\tilde{\zeta}\left(\lambda, T_{1}\right)-\tilde{\zeta}_{\mathrm{F}}\left(\lambda, T_{1}^{\mathrm{F}}\right)\right|<\delta_{1}$;

(3) $\left|\tilde{\zeta}\left(\lambda, T_{2}\right)-\tilde{\zeta}_{\mathrm{B}}\left(\lambda, T_{2}^{\mathrm{B}}\right)\right|<\delta_{2}$;

(4) $\left|\tilde{\zeta}\left(\lambda, T_{3}\right)-\tilde{\zeta}_{\mathrm{B}}\left(\lambda, T_{3}^{\mathrm{B}}\right)\right|<\delta_{3}$;

(5) $\left|\tilde{\zeta}\left(\lambda, T_{4}\right)-X^{+}(\lambda)\right|<\delta_{4}$. 
Part of the proofs of these estimates will be to show that $\tilde{\xi}$ is well defined in each case. To get each estimate will require setting $\varepsilon$ small and the truth of the preceding estimate. The way $\delta_{i}$ depends on $\varepsilon$ is not the same in each case. Each estimate will require a lemma; the proof will then be completed by checking that the estimates can be followed iteratively to reach (5).

There are two different types of lemmas. In the following, $\kappa, \delta_{1}$ and $\delta_{3}$ will be fixed independently of $\varepsilon$. The following two are the first type.

LEMMA 5.5. Given $\delta_{2}>0$, there exists $\varepsilon_{2}>0$ such that if $\varepsilon<\varepsilon_{2}$ then (2) implies (3) for all $\lambda \in \Omega$.

LEMMA 5.6. Given $\delta_{4}>0$, there exists $\varepsilon_{3}>0$ such that if $\varepsilon<\varepsilon_{3}$ then (4) implies (5) for all $\lambda \in \Omega$.

Estimates (2) and (4) are understood to hold for these fixed $\delta_{1}$ and $\delta_{3}$. I shall only prove Lemma 5.5, as 5.6 is similar and easier.

Proof of Lemma 5.5. I shall first set $\kappa$ and $\delta_{1}$. Fix $\kappa_{0}$ so that Lemma 5.3 holds with $\kappa=\kappa_{0}$. According to that lemma there is a neighborhood $Q$ of $K$ in $\left[0, \theta_{2}\right] \times$ $\mathrm{CP}^{2} \times \Omega=F$ so that $\omega(Q) \cap F=K . \theta_{2}$ here depends on $\kappa=\kappa_{0}$; rename it $\theta_{2}^{0}$.

I shall now reset $\kappa$ to be some number smaller than $\kappa_{0}$. Let $\pi_{0}: S^{1} \times \mathrm{CP}^{2} \times \Omega \rightarrow S^{1}$ be the natural projection. Choose $\theta$ so that $\pi_{0}^{-1}(\bar{\theta}) \cap \operatorname{int}(Q) \neq \varnothing$. The first requirement of $\kappa$ is that $J_{\mathrm{F}} \cap B_{1} \subset h_{0}\left(\left[\bar{\theta}, \bar{\theta}_{1}\right]\right)$ and $J_{\mathrm{B}} \cap B_{2} \subset h_{0}\left(\left[\bar{\theta}_{2}, \theta_{2}^{0}\right]\right)$ both be true.

Recalling that $\zeta_{F}(\lambda, \xi)=j\left(\zeta_{R}(\lambda, \xi)\right)$ and noticing that $j\left(X_{1}^{+}(\lambda)\right)=X\left(\bar{\theta}_{1}, \lambda\right)$, it follows from Lemma 5.4 that there exists a $\bar{\xi}$ so that

$$
\left(\theta_{F}(\xi), \hat{\zeta}_{F}(\lambda, \xi), \lambda\right) \in Q
$$

for all $\lambda \in \Omega$ and $\xi \geqslant \bar{\xi}$, where $Q$ remains the attractor neighborhood for the setting $\kappa_{0}$. The time $T_{1}^{\mathrm{F}}$ depends on $\kappa$. Set $\kappa$ so that $T_{1}^{\mathrm{F}} \geqslant \bar{\xi}$. Then $(5.10)$ holds if $\xi=T_{1}^{\mathrm{F}}$ for all $\lambda \in \Omega$. This completes the setting of $\kappa$

To set $\delta_{1}$ I shall need to describe open subsets of $Q$ in local coordinates on $\mathrm{CP}^{2}$. Recall that if $p \neq 0$ in $z=(p, q, r) \in \mathbf{C}^{3},(q / p, r / p)$ form local coordinates on $\mathbf{C P}^{2}$. It is easy to check that if $\varepsilon$ is sufficiently small $\hat{X}(\theta, \lambda)$ lies in such a coordinate patch for all $\theta \in\left[\bar{\theta}_{1}, \theta_{2}\right]$ and $\lambda \in \Omega$. In these coordinates, estimates (1)-(5) could be written with "instead of ${ }^{-}$. It is obvious that the two are equivalent.

Let $N_{\delta}$ be a ball of radius $\delta$ about $\hat{\zeta}_{F}\left(\lambda, T_{1}^{\mathrm{F}}\right)$ in $\mathbf{C P}^{2}$ with the above coordinates. Since $Q$ is open and (5.10) is satisfied, there exists $\delta$ so that $\left\{\theta_{F}\left(T_{1}^{\mathrm{F}}\right)\right\} \times N_{\delta} \times\{\lambda\}$ $\subset Q$ if $\delta=\delta_{1}$ for $\lambda \in \Omega$. Set this to be $\delta_{1}$ for estimate (2).

The fact that $K$ is an attractor, relative to $F$, permits various statements that are true for $\varepsilon=0$ to be perturbed to $\varepsilon>0$. Recall that $H_{0}(t)$ is a flow on $S^{1} \times \mathrm{CP}^{2} \times \Omega$, as is $H_{\varepsilon}(t)$ and, further, the dependence on $\varepsilon$ is continuous.

For $\varepsilon=0$ it is true that, given a neighborhood $R$ of $K$ in $F$ and $Q_{0} \subset Q$, with $Q_{0}$ compact, there exists a $T>0$ so that

$$
H_{0}(T) Q_{0} \subset \operatorname{int}(R) .
$$

(5.11) will perturb to $H_{\varepsilon}(t), \varepsilon>0$ and sufficiently small. So, for the same $Q_{0}$ and $R$,

$$
H_{\varepsilon}(T) Q_{0} \subset \operatorname{int}(R) .
$$


Further, if $R \subset Q$ is a neighborhood of $K$ in $F$, there exists a $T>0$ so that

$$
H_{0}([T, 2 T]) R \cap F \subset \operatorname{int}(R) .
$$

This is equivalent to saying that $R$ is an attractor neighborhood. But then this perturbs to $\varepsilon>0$ also and

$$
H_{\varepsilon}([T, 2 T]) R \cap F \subset \operatorname{int}(R) .
$$

Now determine $R$ by the given $\delta_{2}$. From the proof of Lemma 5.3 the point $\left(\theta_{\mathrm{B}}\left(T_{2}^{F}\right), \hat{\zeta}_{\mathrm{B}}\left(\lambda, T_{2}^{F}\right), \lambda\right) \in K$ for all $\lambda \in \Omega$. Let $M_{\delta}(\lambda)$ be a ball of radius $\delta$ about $\hat{\zeta}_{\mathrm{B}}\left(\lambda, T_{2}^{\mathrm{F}}\right)$ in local coordinates on $\mathrm{CP}^{2}$. Given $\delta_{2}$ choose $R$ so that

$$
R \cap\left\{\theta=\theta_{2}\right\} \subset \bigcup_{\lambda \in \Omega}\left\{\theta_{\mathrm{B}}\left(T_{2}^{\mathrm{F}}\right)\right\} \times M_{\delta} \times\{\lambda\}
$$

for all $\lambda \in \Omega$ if $\delta=\delta_{2} . \delta_{2}$ can be assumed to be small enough so that the above holds.

Estimate (3) will then clearly follow if it can be shown that $\left(\theta_{\varepsilon}\left(T_{2}\right), \hat{\zeta}\left(\lambda, T_{2}\right)\right.$, $\lambda) \in R$. This in turn follows if it can be shown that

$$
\left(\theta_{\varepsilon}(\xi), \hat{\zeta}(\lambda, \xi), \lambda\right) \in R \cup F^{\mathrm{c}}
$$

for all sufficiently large $\xi$, where $F^{\mathrm{c}}=$ complement of $F$.

Now set $Q_{0}$ as $\bigcup_{\lambda \in \Omega}\left\{\theta_{F}\left(T_{1}^{\mathrm{F}}\right)\right\} \times \operatorname{cl}\left(N_{\delta}\right) \times\{\lambda\}$, where $\delta=\delta_{1} / 2$. This is a compact subset of $Q$. Since $\theta_{\varepsilon}\left(T_{1}\right)=\theta_{\mathrm{F}}\left(T_{1}^{\mathrm{F}}\right)$, if $\left(\theta_{\varepsilon}\left(T_{1}\right), \hat{\zeta}\left(\lambda, T_{1}\right), \lambda\right) \in Q_{0}$, then clearly (2) is satisfied.

But then there is an $\hat{\varepsilon}$ so that if $\varepsilon<\hat{\varepsilon}$, (5.12) is true. Further, there is an $\tilde{\varepsilon}$ so that (5.14) holds if $\varepsilon<\tilde{\varepsilon}$. So if $\varepsilon_{2}=\min \{\hat{\varepsilon}, \tilde{\varepsilon}\}$, then, when $\varepsilon<\varepsilon_{2}$, (5.15) holds for sufficiently large $\xi$ and the lemma is proved.

To prove Lemma 5.6 one uses the attractor over the left-hand slow manifold, as remarked before Lemma 5.3. The proof is almost identical and would require setting $\delta_{3}$ and resetting $\kappa$. The following lemmas give the steps from (1) to (2) and (3) to (4).

LEMMA 5.7. Given $\delta_{1}>0$ there exist $\varepsilon_{1}>0$ and $\delta_{0}>0$ so that if $\varepsilon<\varepsilon_{1}$ and (1) is satisfied for all $\lambda \in \Omega$, then (2) also holds for all $\lambda \in \Omega$.

LEMMA 5.8. Given $\delta_{3}>0$ there exist $\varepsilon_{3}>0$ and $\delta_{2}>0$ so that if $\varepsilon<\varepsilon_{3}$ and (3) is satisfied for all $\lambda \in \Omega$, then (4) also holds for all $\lambda \in \Omega$.

Again I shall only prove Lemma 5.7. Lemma 5.8 is the same with the appropriate modification to replace the front with the back.

Proof of Lemma 5.8. The flow $H_{0}(t)$ on $S^{1} \times \mathbf{C P}^{2} \times \Omega$ takes the curve $\left(\theta_{\mathrm{F}}\left(T_{0}^{\mathrm{F}}\right), \hat{\zeta}_{\mathrm{F}}\left(\lambda, T_{0}^{\mathrm{F}}\right), \lambda\right)$, where $\lambda \in \Omega$, in time $T_{1}^{\mathrm{F}}-T_{0}^{\mathrm{F}}$ to the curve of points $\left(\theta_{\mathrm{F}}\left(T_{1}^{\mathrm{F}}\right), \hat{\zeta}_{\mathrm{F}}\left(\lambda, T_{1}^{\mathrm{F}}\right), \lambda\right)$.

If $\kappa$ is small enough, $\hat{\zeta}_{\mathrm{F}}\left(\lambda, T_{i}^{\mathrm{F}}\right), i=0,1$, are both in the usual coordinate patch $(p \neq 0)$. If (1) is satisfied, $\left\{\hat{\zeta}\left(\lambda, T_{0}\right): \lambda \in \Omega\right\}$ lies in a neighborhood of the curve $\left\{\hat{\zeta}_{\mathrm{F}}\left(\lambda, T_{0}^{\mathrm{F}}\right): \lambda \in \Omega\right\}$ of radius $\delta_{0}$.

$T_{0}$ is set so that $\theta_{\varepsilon}\left(T_{0}\right)=\theta_{\mathrm{F}}\left(T_{0}^{\mathrm{F}}\right), T_{1}$ gives $\theta_{\varepsilon}\left(T_{1}\right)=\theta_{\mathrm{F}}\left(T_{1}^{\mathrm{F}}\right)$. Also, from the construction of the pulse, it is not hard to see that $T_{1}(\varepsilon)-T_{0}(\varepsilon) \rightarrow T_{1}^{\mathrm{F}}-T_{0}^{\mathrm{F}}$ as $\varepsilon \rightarrow 0$. 
Therefore by continuity of the flow in $\varepsilon$, if $\varepsilon_{1}, \delta_{0}$ are small enough and $\varepsilon<\varepsilon_{1}$, $\hat{\zeta}\left(\lambda, T_{1}\right)$ lies in a prescribed $\left(\delta_{1}\right)$ neighborhood of $\hat{\zeta}_{\mathrm{F}}\left(\lambda, T_{1}^{\mathrm{F}}\right)$. Estimate (2) then easily follows.

One more ingredient is needed for the proof of Theorem 5.1, that is, that (5) suffices.

Lemma 5.9. There exists $\delta_{4}>0$ so that if (5) is true for $T_{4}$ sufficiently large, uniformly in $\lambda \in \Omega$, then

$$
\operatorname{Re}\left(\tilde{\zeta}\left(\lambda, T_{4}\right) \cdot \tilde{\eta}\left(\lambda, T_{4}\right)\right)>0
$$

for $\lambda \in \Omega$. In particular, such $a \lambda$ is not an eigenvalue.

Proof. First, compute

$$
X^{+} \cdot Y^{-}=1+\alpha^{+} /\left(c-\bar{\beta}^{-}\right)-\varepsilon\left[\left(c-\bar{\beta}^{-}\right)\left(\bar{\beta}^{-}+(\lambda+\varepsilon \alpha) / c\right)\right]^{-1} .
$$

If $\varepsilon=0$ this simplifies to

$$
X^{+} \cdot Y^{-}=1+\alpha^{+} /\left(c-\bar{\beta}^{-}\right) .
$$

One then checks that $\operatorname{Re}\left(\alpha^{+} /\left(c-\bar{\beta}^{-}\right)\right)>0$ and so $\operatorname{Re}\left(X^{+} \cdot Y^{-}\right)>1$.

For the case $\varepsilon>0$ one checks again that $\left(c-\bar{\beta}^{-}\right)\left(\bar{\beta}^{-}+(\lambda+\varepsilon \alpha) / c\right)$ is bounded away from zero, uniformly in $\lambda$ as $\varepsilon \rightarrow 0$. It follows that if $\varepsilon_{0}$ is small enough, then $b$ can be chosen so that there exists $a>0$ for which $\operatorname{Re}\left(X^{+} \cdot Y^{-}\right)>a$ for all $\lambda \in \tilde{G}$ and $\varepsilon \in\left[0, \varepsilon_{0}\right)$.

But $\tilde{\eta}(\lambda, \xi) \rightarrow Y^{-}(\lambda)$ as $\xi \rightarrow+\infty$, and by continuity in $\lambda$ and compactness of $\Omega$, if $T_{4}$ is large enough, $\left|\tilde{\eta}\left(\lambda, T_{4}\right)-Y^{-}\right|$can be made as small as desired uniformly in $\lambda \in \Omega$. The fact that $\operatorname{Re}\left(\tilde{\zeta}\left(\lambda, T_{4}\right) \cdot \tilde{\eta}\left(\lambda, T_{4}\right)\right)<0$ then follows for $\varepsilon \in\left[0, \varepsilon_{0}\right]$ with $\varepsilon_{0}$ sufficiently small, from (5).

Proof of Theorem 5.1. First set $\delta_{1}, \delta_{3}$ and $\kappa$ as required in Lemmas 5.5 and 5.6. Set $\varepsilon_{0}<\varepsilon_{i}, i=1,2,3,4$. Fix $\delta_{4}>0$ so that the conclusion of Lemma 5.9 holds. Proceeding through the estimates, one sees that if $0<\varepsilon<\varepsilon_{0}$, then (1) implies (5), which implies the theorem. It remains to show that (1) holds.

Recall that $\bar{\theta}=0$ at the origin and $X^{+}(\lambda, \varepsilon)$ is the unstable eigenvector for the system (3.5). The point

$$
\left(0, \hat{X}^{+}(\lambda, \varepsilon), \lambda\right)
$$

is then an equilibrium point for all $\lambda \in \Omega$. Arguing in the same fashion as in the proof of Lemma 5.3, the curve $\left(\theta_{\varepsilon}(\xi), \hat{\zeta}(\lambda, \xi), \lambda\right), \xi \in R$, is the unstable manifold $W^{u}$ of (5.17). Since they depend continuously on parameters, as does (5.17), estimate (1) is easily seen to hold, again resetting $\kappa$ if necessary.

6. Winding number computation. From the last section it is known that the only eigenvalues that offer any threat to stability are those near either the front or the back. Since, for both the front and the back, 0 is the eigenvalue of largest real part, any such dangerous eigenvalue must lie close to 0 . In this section I shall prove that there are exactly two eigenvalues near 0 .

Let $B$ be a closed ball of radius $\delta$ about 0 . Set $K=\partial B$. Choose $\delta$ small enough so that

(1) $B \cap\left\{\sigma_{\mathrm{F}} \cup \sigma_{\mathrm{B}}\right\}=\{0\}$, and 
(2) $B \subset \tilde{G}$.

From (2) $\tilde{D}$ is well defined on $K$, for all $\varepsilon \in\left[0, \varepsilon_{0}\right]$, even if $D$ is not.

If $C \subset \mathbf{C} \backslash\{0\}$ is a curve, let $W(C)$ be the usual winding number; i.e., $C$ is given by a function $\phi: S^{1} \rightarrow \mathbf{C} \backslash\{0\} . \phi$ determines an element of $\pi_{1}(\mathbf{C} \backslash\{0\})$, the fundamental group; call it $\pi_{1}(\phi)$. Then $W(C)=\pi_{1}(\phi)$.

Let $\delta$ be as above and choose $\varepsilon_{0}$ so that Theorem 5.1 holds with this $\delta$ if $\varepsilon<\varepsilon_{0}$. In fact, the conclusion of Lemma 5.9, i.e., (5.16), holds for $\lambda \in \Omega$, not just $\lambda \in G_{2}$. In particular, $\tilde{D}(\lambda) \neq 0$ for $\lambda \in K$ so $W(\tilde{D}(K))$ is well defined. The result of this section is the following.

THEOREM 6.1. With $K$ given as above, if $\varepsilon_{0}$ is small enough,

$$
W(\tilde{D}(K))=2 \text {. }
$$

Since $\tilde{D}$ is an analytic function, the winding number counts the number of zeroes of $\tilde{D}$ (by multiplicity) inside $B$. It follows from Theorem 6.1 that there are exactly two zeroes. These zeroes may not correspond to eigenvalues which are zeroes of $D$. However, if there is an unstable eigenvalue, it must be a zero of $D$ and hence a zero of $\tilde{D}$. It would therefore be counted by (6.1).

It is known from the previous section that $\hat{\zeta}(\lambda, \xi)$ can be followed around $S_{\varepsilon}$ and used to show, by its value at large $\xi$, that $\tilde{D}(\lambda) \neq 0$ for all $\lambda \in K$. Information is, however, lost in projectivising and this is insufficient to determine (6.1). The extra information about complex amplitude must be recovered.

Set $\zeta(\lambda, \xi)=(p(\lambda, \xi), q(\lambda, \xi), r(\lambda, \xi))$. As mentioned in $\S 5$, if $\xi=T_{i}, p\left(\lambda, T_{i}\right) \neq$ $0, i=0, \ldots, 4$, for all $\lambda \in K$ (since $K \subset \Omega$ ). This means $\tilde{\zeta}\left(\lambda, T_{i}\right)$ is defined for all such $i$. Define $\gamma_{i}(\lambda)$ for $\lambda \in K, i=0, \ldots, 4$,

$$
\zeta\left(\lambda, T_{i}\right)=\gamma_{i}(\lambda) \tilde{\zeta}\left(\lambda, T_{i}\right) .
$$

In fact, it is obvious that $\gamma_{i}(\lambda)=p\left(\lambda, T_{i}\right)$.

Recall that $\tilde{D}(\lambda)$ is independent of $\xi$ and so can be evaluated at $\xi=T_{4}$. Now,

$$
\tilde{D}(\lambda)=\zeta\left(\lambda, T_{4}\right) \cdot \eta\left(\lambda, T_{4}\right)=\gamma_{4}(\lambda)\left\{\tilde{\zeta}\left(\lambda, T_{4}\right) \cdot \eta\left(\lambda, T_{4}\right)\right\} \text {. }
$$

Also, if $T_{4}$ is large enough,

$$
\exp \left(\beta^{-} T_{4}\right) \eta\left(\lambda, T_{4}\right)=\tilde{\eta}\left(\lambda, T_{4}\right)+\varepsilon\left(\lambda, T_{4}\right),
$$

where

$$
\left|\varepsilon\left(\lambda, T_{4}\right)\right| \rightarrow 0 \text { as } T_{4} \rightarrow+\infty
$$

uniformly for $\lambda \in K$. This follows from the defining condition for $\eta$. Putting this into the expression for $\tilde{D}(\lambda)$,

$$
\tilde{D}(\lambda)=\gamma_{4}(\lambda) \exp \left(\beta^{-} T_{4}\right)\left\{\tilde{\zeta}\left(\lambda, T_{4}\right) \cdot \tilde{\eta}\left(\lambda, T_{4}\right)+\tilde{\zeta}\left(\lambda, T_{4}\right) \cdot \varepsilon\left(\lambda, T_{4}\right)\right\} .
$$

From Lemma 5.9 and (6.3), the term in parentheses has winding number zero. Also if $K$ is small enough, $\bar{\beta}^{-}(\lambda)$ is approximated by $\bar{\beta}^{-}(0)$ for all $\lambda \in K$, so $W\left(\exp \left(\beta^{-}(K) T_{4}\right)\right)=0$. It follows that

$$
W(\tilde{D}(K))=W\left(\gamma_{4}(K)\right) \text {. }
$$


The proof will follow the same style as that of $\$ 5$. I shall iteratively establish the following winding numbers:

(1) $W\left(\gamma_{0}(K)\right)=0$;

(2) $W\left(\gamma_{1}(K)\right)=1$;

(3) $W\left(\gamma_{2}(K)\right)=2$;

(4) $W\left(\gamma_{3}(K)\right)=2$;

(5) $W\left(\gamma_{4}(K)\right)=2$.

The tube parameter $\kappa$ may be reset in the following lemmas, but it will again not depend on $\varepsilon$.

LEMMA 6.1. There exists $\varepsilon_{1}$ so that if $\varepsilon<\varepsilon_{1}$ then $W\left(\gamma_{0}(K)\right)=0$.

Proof. From its definition,

$$
\zeta(\lambda, \xi)=e^{\alpha^{+} \xi} X^{+}+g(\xi),
$$

where $|g(\xi)| \rightarrow 0$ faster than $e^{\left(\operatorname{Re} \alpha^{+}\right) \xi}$. From the proof of Lemma 3.3, this can be made uniform in $\lambda \in K$ and $\varepsilon \in\left[0, \varepsilon_{0}\right]$, i.e., there exists $\nu>0, k$ and $\xi^{*}$ so that

$$
|g(\xi)| \leqslant k e^{\left(\operatorname{Re} \alpha^{+}+\nu\right) \xi}
$$

for all $\xi \leqslant \xi^{*}$ and $\lambda \in K, \varepsilon \in\left[0, \varepsilon_{0}\right]$. Then if $\xi=T_{0}$ is negative enough,

$$
\operatorname{Re} p\left(\lambda, T_{0}\right)>0
$$

for all $\lambda \in K$ and $\varepsilon \in\left[0, \varepsilon_{0}\right]$. It is clear that $\kappa$ can be reset so that the $T_{0}$ satisfying $\theta_{\varepsilon}\left(T_{0}\right)=b_{0}$ is negative enough for all $\varepsilon \in\left[0, \varepsilon_{0}\right]$.

From (6.4), since $\gamma_{0}(\lambda)=p\left(\lambda, T_{0}\right)$, (1) easily follows.

LEMMA 6.2. There exists $\varepsilon_{2}$ so that if $\varepsilon<\varepsilon_{2}$ then $W\left(\gamma_{1}(K)\right)=1$.

Proof. First consider the behavior of the reduced system, i.e., the front. Recall the definitions of $T_{0}^{\mathrm{F}}$ and $T_{1}^{\mathrm{F}}$ and set

$$
\zeta_{\mathrm{F}}\left(\lambda, T^{\mathrm{F}}\right)=\gamma_{1}^{\mathrm{F}}(\lambda) \tilde{\zeta}_{\mathrm{F}}\left(\lambda, T_{1}^{\mathrm{F}}\right) ;
$$

as usual, this is well defined. Now

$$
D_{\mathrm{F}}(\lambda)=\zeta_{\mathrm{F}}\left(\lambda, T_{1}^{\mathrm{F}}\right) \cdot \eta_{\mathrm{F}}\left(\lambda, T_{1}^{\mathrm{F}}\right)=\gamma_{1}^{\mathrm{F}}(\lambda)\left\{\tilde{\zeta}_{\mathrm{F}}\left(\lambda, T_{1}^{\mathrm{F}}\right) \cdot \eta\left(\lambda, T_{1}^{\mathrm{F}}\right)\right\} .
$$

From the same kind of argument as given earlier, and using Lemma 5.4,

$$
W\left(\tilde{\zeta}_{\mathrm{F}}\left(K, T_{1}^{\mathrm{F}}\right) \cdot \eta\left(K, T_{1}^{\mathrm{F}}\right)\right)=0
$$

if $K$ is small enough ( $K$ determines $T_{1}^{\mathrm{F}}$ ). But then

$$
W\left(D_{\mathrm{F}}(K)\right)=W\left(\gamma_{1}^{\mathrm{F}}(K)\right) .
$$

From Lemma $4.1,\left.(d / d \lambda) D_{F}(\lambda)\right|_{\lambda=0}>0$. If $\delta$ is small enough (resetting $\varepsilon_{0}$ if necessary), $K$ will be a small circle about 0 . It follows that

$$
W\left(D_{\mathrm{F}}(K)\right)=1 \text { and } W\left(\gamma_{1}^{\mathrm{F}}(K)\right)=1 .
$$

This is where the stability of the front is used.

I shall translate this information into a map. Let

$$
C_{0}^{\mathrm{F}}(\lambda)=\operatorname{span}_{\mathbf{c}}\left\{\zeta_{\mathrm{F}}\left(\lambda, T_{0}^{\mathrm{F}}\right)\right\}
$$


and

$$
E_{0}^{\mathrm{F}}=\left\{(w, \lambda): w \in C_{0}^{\mathrm{F}}(\lambda), \lambda \in K\right\} .
$$

Put coordinates on $E_{0}^{\mathrm{F}}$ by using the map

$$
\mathrm{C} \times K \rightarrow E_{0}^{\mathrm{F}}, \quad(z, \lambda) \mapsto\left(z \tilde{\zeta}_{\mathrm{F}}\left(\lambda, T_{0}^{\mathrm{F}}\right), \lambda\right) .
$$

If $(z, \lambda) \in E_{0}^{\mathrm{F}}$, take $z \tilde{\zeta}_{\mathrm{F}}\left(\lambda, T_{0}^{\mathrm{F}}\right)$ as an initial condition for the eigenvalue flow, determined by (4.4), at time $T_{0}^{\mathrm{F}}$. Following this, for each $\lambda$ up until time $T_{1}^{\mathrm{F}}$, one obtains a multiple of $\tilde{\zeta}_{\mathrm{F}}\left(\lambda, T_{1}^{\mathrm{F}}\right)$; call this $\phi^{\mathrm{F}}(z, \lambda) \tilde{\zeta}_{\mathrm{F}}\left(\lambda, T_{1}\right)$. So $\phi$ is a map $\phi^{\mathrm{F}}$ : $E_{0}^{\mathrm{F}} \rightarrow \mathrm{C}$. Since the flow is linear, $\phi$ is linear in $z$. Set $\phi^{\mathrm{F}}(z, \lambda)=\Phi^{\mathrm{F}}(\lambda) z$. $\Phi^{\mathrm{F}}$ is now a map $\Phi^{\mathrm{F}}: K \rightarrow \mathrm{C} \backslash\{0\}$. It is easy to check that $\Phi^{\mathrm{F}}$ is continuous.

Now evaluate $\phi^{\mathrm{F}}$ on $\gamma_{1}^{\mathrm{F}}(\lambda)$ :

$$
\phi^{\mathrm{F}}\left(\gamma_{1}^{\mathrm{F}}(\lambda), \lambda\right)=\Phi^{\mathrm{F}}(\lambda) \gamma_{1}^{\mathrm{F}}(\lambda)
$$

But from the definition of $\phi^{\mathrm{F}}$ it is easy to see that

$$
\phi^{\mathrm{F}}\left(\gamma_{1}^{\mathrm{F}}(\lambda), \lambda\right)=\gamma_{1}^{\mathrm{F}}(\lambda)
$$

Consequently,

$$
\Phi^{\mathrm{F}}(\lambda)=\gamma_{2}^{\mathrm{F}}(\lambda) / \gamma_{1}^{\mathrm{F}}(\lambda) .
$$

Now approximate the $\varepsilon \neq 0$ case by the reduced system. Consider the eigenvalue system (3.3) again. Let $y(\lambda, \xi)$ be the solution of (3.3) satisfying the condition $y\left(\lambda, T_{0}\right)=\tilde{\zeta}\left(\lambda, T_{0}\right)$, which obviously depends on $\varepsilon$. Let $y_{\mathrm{F}}(\lambda, \xi)$ be the solution of the reduced system (4.4) (with $r^{\prime}=0$ appended) satisfying

$$
y_{\mathrm{F}}\left(\lambda, T_{0}^{\mathrm{F}}\right)=\tilde{\zeta}_{\mathrm{F}}\left(\lambda, T_{0}^{\mathrm{F}}\right) \text {. }
$$

Because $\theta_{\varepsilon}\left(T_{0}\right)=b_{0}=\theta_{\mathrm{F}}\left(T_{0}^{\mathrm{F}}\right)$ and (5.1) is continuous, $\left|y(\lambda, T)-y_{\mathrm{F}}(\lambda, T)\right|$ can be made as small as desired for fixed $T$ (if $\varepsilon$ is small enough). But also $T_{1}-T_{0} \rightarrow$ $T_{1}^{\mathrm{F}}-T_{0}^{\mathrm{F}}$ as $\varepsilon \rightarrow 0$. It follows that if $\nu$ is prescribed, there is an $\varepsilon$, so that $\varepsilon<\varepsilon_{1}$ implies

$$
\left|y\left(\lambda, T_{1}\right)-y_{\mathrm{F}}\left(\lambda, T_{1}^{\mathrm{F}}\right)\right|<\nu .
$$

Let $C_{0}(\lambda)=\operatorname{span}_{\mathrm{C}}\left\{\zeta\left(\lambda, T_{0}\right)\right\}$ and $E_{0}=\left\{(\omega, \lambda): \omega \in C_{0}(\lambda), \lambda \in K\right\}$. Just as before let $(z, \lambda)$ be coordinates on $E_{0}$ where $w=z \tilde{\zeta}\left(\lambda, T_{0}\right)$. By taking $z \tilde{\zeta}\left(\lambda, T_{0}\right)$ as the initial condition again, define $\phi(z, \lambda)$ by requiring that $\phi(z, \lambda) \tilde{\zeta}\left(\lambda, T_{1}\right)$ be the solution at time $T_{1}$. Again $\phi$ is linear in $z$. Set $\phi(z, \lambda)=\Phi(\lambda) z$. Also as above,

$$
\Phi(\lambda)=\gamma_{2}(\lambda) / \gamma_{1}(\lambda) \text {. }
$$

$\Phi(\lambda) 1=\phi(1, \lambda)=$ the first component of $y\left(\lambda, T_{1}\right)$. Also $\Phi^{\mathrm{F}}(\lambda)=$ the first component of $y_{\mathrm{F}}\left(\lambda, T_{1}^{\mathrm{F}}\right)$. But then, by (6.4), $\left|\gamma_{1}(\lambda) / \gamma_{0}(\lambda)-\gamma_{1}^{\mathrm{F}}(\lambda) / \gamma_{0}^{\mathrm{F}}(\lambda)\right|$ can be made as small as desired uniformly in $\lambda \in K$. Therefore, $\gamma_{1}(\lambda)-\left\{\gamma_{1}^{\mathrm{F}}(\lambda) / \gamma_{0}^{\mathrm{F}}(\lambda)\right\} \gamma_{0}(\lambda)$ can also be made small. But then

$$
W\left(\gamma_{1}(K)\right)=W\left(\gamma_{1}^{\mathrm{F}}(K) / \gamma_{0}^{\mathrm{F}}(K)\right)+W\left(\gamma_{0}(K)\right) .
$$

From Lemma 6.1 and the above arguments it follows that $W\left(\gamma_{1}(K)\right)=1+0=1$.

LEMMA 6.3. If $\varepsilon<\varepsilon_{0}$ (as determined in $\left.§ 5\right)$ then $W\left(\gamma_{2}(K)\right)=1$. 
Proof. If $\varepsilon<\varepsilon_{0}$, from the proof of Theorem 5.1, if $\zeta=(p, q, r)$, then $p(\lambda, \xi) \neq 0$ for all $\lambda \in K$ and $T_{1} \leqslant \xi \leqslant T_{2}$. Since $\gamma_{1}(\lambda)=p\left(\lambda, T_{1}\right)$ and $\gamma_{2}(\lambda)=p\left(\lambda, T_{2}\right), p(\lambda, \xi)$ defines a homotopy of $\gamma_{1}: K \rightarrow \mathbf{C} \backslash\{0\}$ to $\gamma_{2}: K \rightarrow \mathbf{C} \backslash\{0\}$. Therefore $W\left(\gamma_{2}(K)\right)=$ $W\left(\gamma_{1}(K)\right)$ and the lemma is proved.

LEMMA 6.4. There exists $\varepsilon_{2}$ so that if $\varepsilon<\varepsilon_{2}$ then $W\left(\gamma_{3}(K)\right)=2$.

Proof. The analysis closely follows that of Lemma 6.2, but the back is used to approximate instead of the front. The conclusion is that

$$
W\left(\gamma_{3}(K)\right)=W\left(\gamma_{2}(K)\right)+1=2 .
$$

LEMMA 6.5. If $\varepsilon<\varepsilon_{0}$ then $W\left(\gamma_{4}(K)\right)=2$.

Proof. $p(\lambda, \xi)$ gives a homotopy, just as in the case of Lemma 6.3. Therefore, $W\left(\gamma_{4}(K)\right)=W\left(\gamma_{3}(K)\right)=2$.

This completes the proof of Theorem 6.1.

7. Completion of proof. From Lemma 3.2, if $\varepsilon$ is small, the essential spectrum $\sigma_{\mathrm{e}}(L)$ lies entirely in a set $\{\lambda: \operatorname{Re} \lambda<a\}$, where $a<0$, albeit dependent on $\varepsilon$. Therefore it is only eigenvalues that can cause instability. From Theorem 5.1 these eigenvalues must lie in a $\delta$-neighborhood of 0 , where $\delta=\delta(\varepsilon) \rightarrow 0$ as $\varepsilon \rightarrow 0$.

Theorem 6.1 says that there are two zeroes of $D$ in such a $\delta$-neighborhood. Therefore there are at most two zeroes of $D$, and so there are at most two eigenvalues. Since 0 is definitely an eigenvalue (due to translation) the other zero of $D$ must be real.

If $\lambda>0$ and large it dominates the system (3.3)-(3.4). It is not hard to check that $D(\lambda)>0$ in this situation; see Evans [9] for details. It would follow that the other zero of $D(\lambda)$ near zero is negative if it could be established that

$$
\left.(d / d \lambda) D(\lambda)\right|_{\lambda=0}>0 .
$$

The main theorem will follow by proving (7.1).

Evans devised a beautiful technique for computing the sign of $\left.(d / d \lambda) D(\lambda)\right|_{\lambda-0}$ :

$$
\frac{d}{d \lambda} D(\lambda)=\left\{\frac{\partial}{\partial \lambda} \zeta(\lambda, \xi)\right\} \cdot \eta(\lambda, \xi)+\zeta(\lambda, \xi) \cdot\left\{\frac{\partial}{\partial \lambda} \eta(\lambda, \xi)\right\} .
$$

Furthermore, the right-hand side can be evaluated at any $\xi$. As $\xi \rightarrow+\infty,\left|\eta_{\lambda}(0, \xi)\right|$ $\rightarrow 0$ and $|\zeta(0, \xi)| \rightarrow 0$ since $\eta$ is determined at $+\infty$ and $\zeta(0, \xi)$ is the derivative of the travelling wave. Therefore

$$
\left.\frac{d}{d \lambda} D(\lambda)\right|_{\lambda=0}=\left.\lim _{\xi \rightarrow+\infty} \zeta_{\lambda}(\lambda, \xi) \cdot \eta(\lambda, \xi)\right|_{\lambda=0}
$$

$\zeta(\lambda, \xi)=(p(\lambda, \xi), q(\lambda, \xi), r(\lambda, \xi))$ satisfies system (3.2). Differentiating with respect to $\lambda$ and evaluating at $\lambda=0, \zeta_{\lambda}(0, \xi)$ satisfies

$$
\begin{gathered}
x^{\prime}=y, \quad y^{\prime}=-c y-f^{\prime}(u) x+z+p(0, \xi), \\
z^{\prime}=-(\varepsilon / c) x+(\varepsilon \gamma / c) z+(1 / c) r(0, \xi) .
\end{gathered}
$$


Fix $\varepsilon \in\left(0, \varepsilon_{0}\right]$. Let $\bar{c}(\varepsilon)$ be the speed at which the pulse exists. For each $c$ in a neighborhood of $\bar{c}(\varepsilon)$, there exists a solution (unique up to parametrisation) that tends to 0 as $\xi \rightarrow-\infty$. Call this $U(c, \xi)=(u(c, \xi), v(c, \xi), w(c, \xi))$, with parametrisation set by $u(c, 0)=a$ with no $\xi<0$ satisfying $u(c, \xi)=a$ (recall $a$ is the middle zero of $f(u)$ ). This condition uniquely determines $U(c, \xi)$, if $\varepsilon_{0}$ is small enough. Note that $U(c, \xi)$ is the unstable manifold of the critical point $(0,0,0)$.

Differentiating the travelling wave system (2.1) with respect to $c$ and evaluating at $c=\bar{c}(\varepsilon), U_{c}$ satisfies

$$
\begin{gathered}
x^{\prime}=y, \quad y^{\prime}=-c y-f^{\prime}(u) x+z-u_{\xi}, \\
z^{\prime}=-(\varepsilon / c) x+(\varepsilon \gamma / c) z-(1 / c) w_{\xi} .
\end{gathered}
$$

Now by definition, $(p(0, \xi), q(0, \xi), r(0, \xi))$ satisfies system (3.2) with $\lambda=0$, which is

$$
p^{\prime}=q, \quad q^{\prime}=-c q-f^{\prime}(u) p+r, \quad r^{\prime}=-(\varepsilon / c) p+(\varepsilon \gamma / c) r .
$$

It is easily seen that if $(u(\xi), v(\xi), w(\xi))$ is the travelling wave then $\left(u_{\xi}, v_{\xi}, w_{\xi}\right)$ also satisfies (7.4) with $c=\bar{c}(\varepsilon)$. The solution of (7.4) that decays to 0 as $\xi \rightarrow-\infty$ is unique up to a scalar multiple. So there is a scalar $\alpha$ for which $\alpha p(0, \xi)=u_{\xi}(\xi)$ and $\alpha r(0, \xi)=w_{\xi}(\xi)$. From (7.2) and (7.3), $\alpha \zeta_{\lambda}+U_{c}$ must then satisfy (7.4), but the only solution of (7.4) which decays to 0 as $\xi \rightarrow-\infty$ is $\zeta(0, \xi)$ itself. Since $\alpha \zeta_{\lambda}+U_{c}$. clearly does, there is a $b$ so that $\alpha \zeta_{\lambda}+U_{c}=b \zeta$.

Moreover, $\alpha$ must be greater than 0 , since $p(0, \xi)>0$ for large negative $\xi$; it is asymptotic to $X^{+}$, whose first component is 1 ; and $u_{\xi}>0$ for large negative $\xi$.

Substituting into the above expression,

$$
\begin{aligned}
\left.\frac{d}{d \lambda} D(\lambda)\right|_{\lambda=0} & =\lim _{\xi \rightarrow+\infty}\left(\frac{b}{\alpha} \zeta-\frac{1}{\alpha} U_{c}\right) \cdot \eta(0, \xi) \\
& =\lim _{\xi \rightarrow+\infty}\left(\frac{b}{\alpha} \zeta \cdot \eta-\frac{1}{\alpha} U_{c} \cdot \eta\right)=-\frac{1}{\alpha} \lim _{\zeta \rightarrow+\infty} U_{c} \cdot \eta .
\end{aligned}
$$

The last equality holds because $\zeta \cdot \eta=0$, which is true at $\lambda=0$ since it is an eigenvalue.

As noted in $\S 3, \eta(0, \xi)$ is normal to the stable subspace of $(0,0,0)$. Therefore, the limit on the right-hand side contains information about how the solution $U$ crosses this subspace with respect to $c$. In other words, its sign is determined by the direction in which the unstable manifold crosses the stable manifold, with respect to $c$, at the value of $c$ for which the wave exists.

Unfortunately the quantity $U_{c} \cdot \eta$ is not independent of $\xi$ and so the limit cannot be dropped in (7.5). I shall determine quantities $P(\xi)$ and $N(\xi)$ for which

$$
\lim _{\xi \rightarrow+\infty} P(\xi) \cdot N(\xi)=\lim _{\xi \rightarrow+\infty} U_{c} \cdot \eta,
$$

but $P(\xi) \cdot N(\xi)$ will be independent of $\xi$ and so can be evaluated anywhere along the pulse solution.

Append $c^{\prime}=0$ to the travelling wave system to obtain the system in $\mathbf{R}^{4}$ :

$$
u^{\prime}=v, \quad v^{\prime}=-c v-f(u)+w, \quad w^{\prime}=-(\varepsilon / c)(u-\gamma w), \quad c^{\prime}=0 .
$$


The point $(0,0,0, \bar{c}(\varepsilon))$ is a critical point of $(7.6)$. Let $W^{\text {cu }}(\varepsilon)$ be the center-unstable manifold of this point for (7.6). This is obtained locally and then iterated in forward time. Let $W^{\text {cs }}(\varepsilon)$ be the center-stable manifold obtained in a similar fashion.

If $c$ is close to $\bar{c}(\varepsilon),(U(c, \xi), c)$ lies in $W^{\text {cu }}(\varepsilon)$. Set

$$
P(\xi)=\left(\frac{\partial U}{\partial c}(\bar{c}(\varepsilon), \xi), 1\right) \text {. }
$$

This is tangent to the above curve at the point $(U(\bar{c}(\varepsilon), \xi), \bar{c}(\varepsilon))$. As such, it satisfies the equation of variations for (7.6):

$$
\begin{aligned}
& p^{\prime}=q, \quad q^{\prime}=-c q-f^{\prime}(u) p+r-v s, \\
& r^{\prime}=-(\varepsilon / c)(p-\gamma w) s, \quad s^{\prime}=0,
\end{aligned}
$$

where $u=u(\bar{c}(\varepsilon), \xi)$.

To set $N(\xi)$, rewrite (7.7) as

$$
x^{\prime}=A x
$$

and let

$$
y^{\prime}=B y
$$

be the adjoint system $\left(B=-A^{*}\right) . N(\xi)$ will be a solution of (7.9).

$A$ and $B$ both depend on $\xi$. Let $A_{0}=\lim _{\xi \rightarrow+\infty} A$ and $B_{0}=\lim _{\xi \rightarrow+\infty} B$. With $\varepsilon \neq 0$ there is only one eigenvalue of $A_{0}$ with positive real part. Therefore $B_{0}$ has only one of negative real part. The usual argument shows that there is a unique solution of (7.9), call it $N(\xi)$, up to a scalar multiple, that decays at $+\infty$. It is not hard to convince oneself that this solution is normal to $W^{\text {cs }}(\varepsilon)$ at $(U(\bar{c}(\varepsilon), \xi), \bar{c}(\varepsilon))$ for each $\xi$. Writing out $A$ and taking the adjoint,

$$
B=\left(\begin{array}{cccc}
0 & f^{\prime}(u) & \varepsilon / c & 0 \\
-1 & c & 0 & 0 \\
0 & -1 & -\varepsilon \gamma / c & 0 \\
0 & v & -\left(\varepsilon / c^{2}\right)(u-\gamma w) & 0
\end{array}\right)
$$

From the form of $B$, the first three equations of (7.9) uncouple from the fourth. Therefore these first three equations are the same as those satisfied by $\eta(0, \xi) . N(\xi)$ must be a scalar multiple of $(\eta(0, \xi), k(\xi))$ for some function $k(\xi)$ found by solving the fourth equation of (7.10); this is because $\eta(0, \xi)$ decays to 0 as to $\xi \rightarrow+\infty$. Set $N(\xi)=(\eta(0, \xi), k(\xi))$.

$P(\xi) \cdot N(\xi)$ can now be computed:

$$
P(\xi) \cdot N(\xi)=\frac{\partial U}{\partial c}(\bar{c}(\varepsilon), \xi) \cdot \eta(0, \xi)+k(\xi) .
$$

Since $|N(\xi)| \rightarrow 0$ as $\xi \rightarrow+\infty, k(\xi) \rightarrow 0$ as $\xi \rightarrow+\infty$. Therefore,

$$
\lim _{\xi \rightarrow+\infty} P(\xi) \cdot N(\xi)=\lim _{\xi \rightarrow+\infty} \frac{\partial U}{\partial c}(\bar{c}(\varepsilon), \xi) \cdot \eta(0, \xi) .
$$

Since $P(\xi)$ satisfies (7.8) and $N(\xi)$ satisfies (7.9), $P(\xi) \cdot N(\xi)$ is actually independent of $\xi$, using the same argument as the one which shows $D(\lambda)$ is independent of 
६. Therefore,

$$
P(\tau) \cdot N(\tau)=\lim _{\xi \rightarrow+\infty} U_{c} \cdot \eta
$$

for any $\tau \in \mathbf{R}$.

The theorem will then be proved by finding a $T$ for which

$$
P(T) \cdot N(T)<0 \text {. }
$$

From (7.11) and (7.5), it then follows that $\left.(d / d \lambda) D(\lambda)\right|_{\lambda=0}>0$, as desired.

The proof of (7.12) will require the transversality in Langer's proof [20]. To explain and summarize what is needed from Langer's work, I shall first give some notation.

Consider again the travelling wave system (7.6) in $\mathbf{R}^{4}$. Now set $\varepsilon=0$ and $c=\bar{c}(0)=c^{*}$ (in the notation of \$2). Recall from $\$ 2$ (see Figure 1) that $E_{\mathrm{R}}^{*}$ and $E_{\mathrm{L}}^{*}$ are the parts of the right and left slow manifolds that partake in the singular solutions. Let $E_{\mathrm{R}}$ and $E_{\mathrm{L}}$ be extensions of these in the $w$ directions with $c=c^{*}$, i.e.,

$$
E_{\mathrm{R}}=\left\{(u, v, w, c): v=0, w_{1} \leqslant w \leqslant w_{2}, c=c^{*} \text { is the largest root of } w=f(u)\right\},
$$
where $w_{1}<0$ and $w_{2}>w^{*}$ are suitably chosen; similarly for $E_{\mathrm{L}}$. Let $R^{u}$ be the center-unstable manifold of this curve of critical points; see Fenichel [10]. This is a three-dimensional object. Let $L^{\text {s }}$ be the stable manifold of the left-hand slow manifold $E_{\mathrm{L}}$, lying in the slice $c=c^{*}$. As usual, each of these is obtained locally and then iterated in the appropriate time direction.

Let $\omega=\omega(0)$ be the point in $\mathbf{R}^{3}$ where $J_{B}$ (the back) intersects $\{u=a\}$; recall that $a$ is the middle zero of $f$. Set $K_{0}$ to be the unit normal to $L^{s}$ at $(\omega(0), \bar{c}(0))$ with positive $v$ component. The following argument shows that this is well defined. As $L^{\mathrm{s}}$ is carried in backward time along $J_{\mathrm{B}}$, the sign of the second component of the normal cannot change. With $\varepsilon=0$, one can find two vectors, $v_{1}(\xi)$ and $v_{2}(\xi)$, tangent to $L^{\mathrm{s}}$ at a given point. $v_{1}=\left(p_{1}, q_{1}, r_{1}\right)$ and $v_{2}=\left(p_{2}, q_{2}, r_{2}\right) . v_{1}$ is tangent to $J_{\mathrm{B}}$ with $p_{1}>0$ and $r_{1}=0 . v_{2}$ is not tangent to $J_{\mathrm{B}}$ but has $r_{2}>0$; this can be found because $w=$ constant are invariant planes. But then any normal to $L^{\mathrm{s}}$ at a point on $J_{\mathrm{B}}$ must be a multiple of $v_{1} \times v_{2}$ and, from the above properties, could not have a zero second component.

The set $R^{u} \cap\{c=\bar{c}(0)\} \cap\{u=a\}$ is a curve near $(\omega(0), \bar{c}(0))$. Set $Q_{0}$ to be the unit tangent vector to this curve at $(\omega(0), \bar{c}(0))$ with negative $w$ component. The argument that this is well defined is very similar to that for $K_{0} ; w$ replaces $v$ because this is a tangent not a normal vector.

To define $K_{\varepsilon}$ and $Q_{e}, \varepsilon \neq 0$, let $\omega(\varepsilon)$ be the point on the back of the pulse $S_{\varepsilon}$ in $\{u=a\}$ for small $\varepsilon . K_{\varepsilon}$ is then the unit normal to $W^{c s}(\varepsilon)$ at $(\omega(\varepsilon), \bar{c}(\varepsilon))$, again with positive $v$-component. Let $Q$, be the unit tangent vector to the curve $W^{\text {cu }}(\varepsilon) \cap\{u=$ $a$ ) with negative $w$ component. These are both well defined because they converge to $K_{0}$ and $Q_{0}$, respectively; see below.

With these definitions it is not hard to see that $K_{\varepsilon} \rightarrow K_{0}$ as $\varepsilon \rightarrow 0$. Append yet another equation to (7.6), namely $\varepsilon^{\prime}=0$. Embed $E_{\mathrm{L}}$ into the $\varepsilon=0$ subspace of $\mathbf{R}^{5}$ and consider $W^{\text {cs }}$, the center-stable manifold of $E_{\mathrm{L}}$, now with $\varepsilon$ varying. $W^{\text {cs }} \cap\{\varepsilon=$ $0\} \cap\left\{c=c^{*}\right\}$ and $W^{\text {cs }} \cap\{\varepsilon=\tilde{\varepsilon}\}=W^{c s}(\tilde{\varepsilon})$, the center-stable manifold of the curve of critical points $(0,0,0, c, \tilde{\varepsilon}), c$ varying. These then vary smoothly in $\varepsilon$ since they are 
slices of a smooth manifold. It follows that appropriately oriented normals vary continuously and so $K_{\varepsilon} \rightarrow K_{0}$ as $\varepsilon \rightarrow 0$.

It is considerably harder to see that $Q_{\varepsilon} \rightarrow Q_{0}$ as $\varepsilon \rightarrow 0$, since their definitions are very different. Indeed, that this is true is the hardest part of Langer's proof. The fact that $Q_{\varepsilon}$ lies close to $Q_{0}$ carries information to the back about how the front is constructed.

From Langer's work I shall need, then, the following two facts, which I state as lemmas.

LEMMA 7.1. $Q_{0} \cdot K_{0}<0$.

LEMMA 7.2. $Q_{\varepsilon} \rightarrow Q_{0}$.

Lemma 7.1 is the heart of the matter. Everything else is just designed to see that this is the correct quantity to compute. I shall leave the proof of Lemma 7.1 and its geometric explanation to the end.

I shall proceed by showing how to deduce Lemma 7.2 from Langer's construction and then prove (Lemma 7.3) that the above is what is needed.

Proof of Lemma 7.2. Langer constructs a box $B_{\varepsilon}$ about the right-hand slow manifold in $\mathbf{R}^{4}$ and then considers the intersection of various unstable manifolds with the face $F$ on the boundary of the box that is near the exit point on $J_{\mathrm{B}}$. These are

$$
\alpha_{0}=R^{u} \cap\{c=\bar{c}(0)\} \cap F, \quad \alpha_{\varepsilon}=W^{\text {cu }}(\varepsilon) \cap F .
$$

He then proves that $\alpha_{\varepsilon}$ is close $(O(\varepsilon))$ in the $C^{1}$ topology to $\alpha_{0}$. Let $\pi_{0}$ be the point in $J_{B} \times\{\bar{c}(0)\} \cap F$. The travelling wave system (7.6) in $\mathbf{R}^{4}$ with $\varepsilon^{\prime}=0$ appended induces a smooth flow on $\mathbf{R}^{5}$, and, on some small neighborhood $V$ of $\pi_{0} \times\left[0, \varepsilon_{0}\right)$, it induces a $C^{1}$ diffeomorphism into the set $\{u=a\}$. Call this map $\psi: V \rightarrow\{u=a\}$. It obviously takes $\alpha_{\varepsilon} \times\{\varepsilon\}$ into $\left(W^{\text {cu }}(\varepsilon) \cap\{u=a\}\right) \times\{\varepsilon\}$ and $\alpha_{0} \times\{0\}$ into $\left(R^{u} \cap\{c=\bar{c}(0)\} \cap\{u=a\}\right) \times\{0\}$. These curves therefore remain $C^{1}$-close. Since $Q_{\varepsilon}$ and $Q_{0}$ are unit tangent vectors to these curves at $(\omega(\varepsilon), \bar{c}(\varepsilon))$ and $(\omega(0), \bar{c}(0))$, respectively, $Q_{\varepsilon} \rightarrow Q_{0}$ as $\varepsilon \rightarrow 0$. This proves the lemma.

REMARK. Langer's proof that $\alpha_{\varepsilon}$ is close to $\alpha_{0}$ assumes that $\gamma=0$. However, I claim that it is merely a technical modification to include the case $\gamma \neq 0$.

LeMma 7.3. $\operatorname{sgn}\left(Q_{0} \cdot K_{0}\right)=\operatorname{sgn}(P(T) \cdot N(T))$ for small enough $\varepsilon$, where $T=T(\varepsilon)$ and $U(\bar{c}(\varepsilon), T)=\omega(\varepsilon)$.

Proof. $W^{\text {cu }}(\varepsilon) \cap\{u=a\}$ is a curve and can be parametrised by $c$. Moreover, there is a smooth function $\tau(c)$ so that it is given by $(U(c, \tau(c)), c)$ near $(\omega(\varepsilon), \bar{c}(\varepsilon))$. A tangent vector can be found by differentiating, with respect to $c$,

$$
\left(\partial U / \partial c+(\partial U / \partial \xi) \tau^{\prime}, 1\right)
$$

evaluated at $\bar{c}(\varepsilon)$. It follows that $Q_{\varepsilon}$ is a scalar multiple of (7.13):

$$
Q_{\varepsilon}=m\left(\partial U / \partial c+(\partial U / \partial \xi) \tau^{\prime}, 1\right)
$$


To check the sign of $m$, we must check the sign of $\partial w / \partial c=\tau^{\prime}(\partial w / \partial \xi)$. Now $\tau(c)$ satisfies $u(c, \tau(c))=a$, and so $\tau^{\prime}=-\left(u_{c} / u_{\xi}\right)$. So we want the sign of

$$
w_{c}-u_{c}\left(w_{\xi} / u_{\xi}\right) \text {. }
$$

Langer proves that if $u_{c}$ is evaluated at a point near the right-hand slow manifold there is a $k, \alpha$ so that

$$
\left|u_{c}(\xi)\right| \geqslant k e^{\alpha \xi} \text {. }
$$

I need to recover the sign of $u_{c}$. This information lies in the behavior of the front as $c$ varies. Set $w=0, \varepsilon=0$; the phase portrait for $c=\bar{c}(0)$ is given in Figure 7. For $c>\bar{c}(0)$ but close to it, the phase portrait is that in Figure 8. So when $\varepsilon=0$ and $\xi$ is large, $u_{c} \ll 0$. By continuity it therefore follows for $c=\bar{c}(\varepsilon)$ and $\varepsilon \neq 0$. As $u(\bar{c}(\varepsilon), \xi)$ remains near the right-hand slow manifold, this does not change. Therefore $u_{c}(\xi) \leqslant$ $-k e^{\alpha \xi}$.

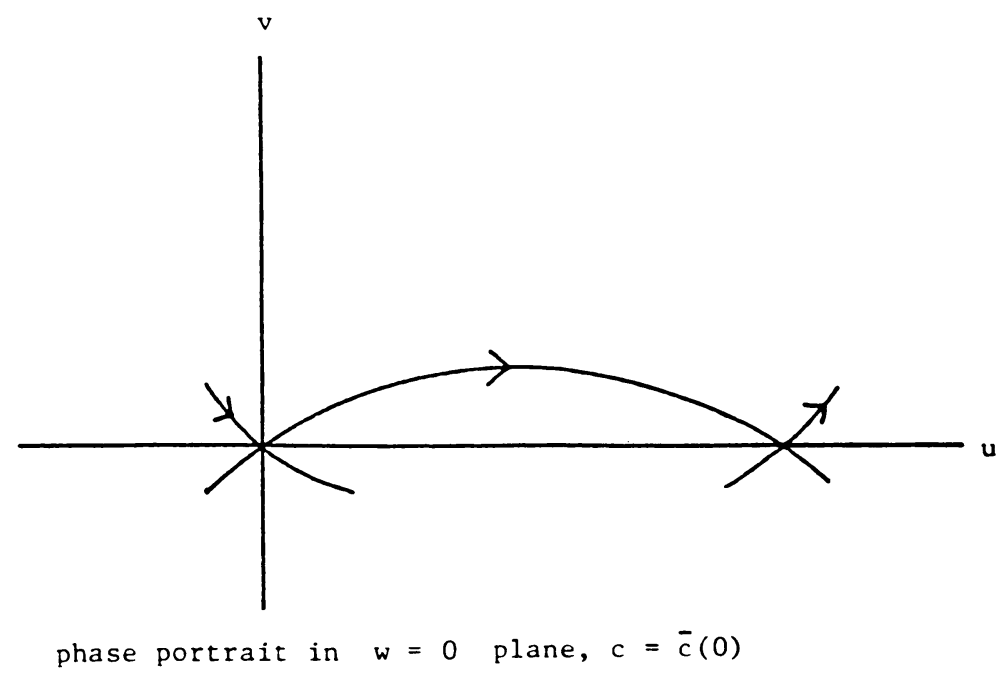

Figure 7

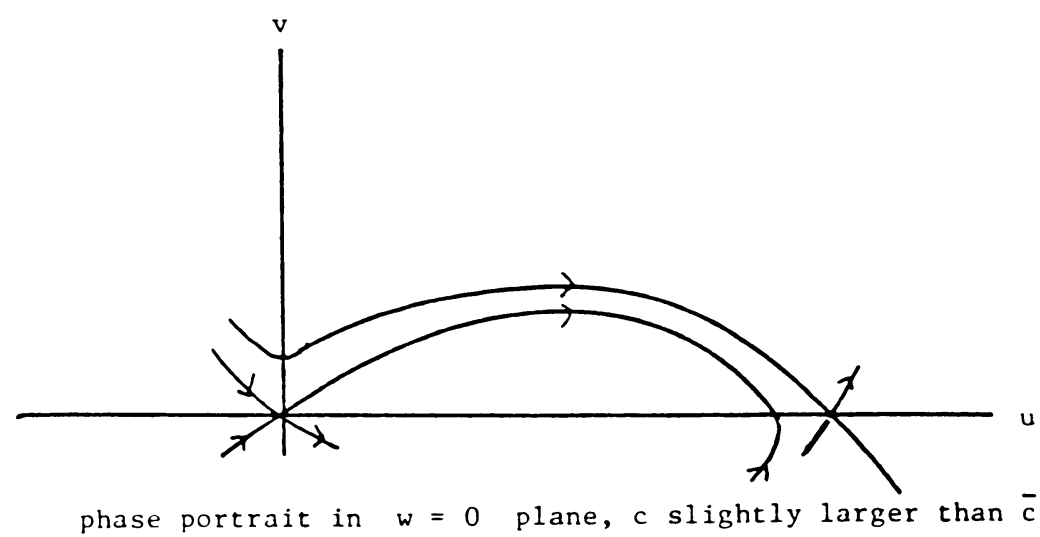

Figure 8 
The above described feature is, in fact, one of the main ingredients in proving $\alpha_{\varepsilon}$ is close to $\alpha_{0}$.

The equation for $w_{c}$ is

$$
w_{c}^{\prime}=-(\varepsilon / c)\left(u_{c}-\gamma w_{c}\right)+\left(\varepsilon / c^{2}\right)(u-\gamma w) .
$$

Near $E_{\mathrm{L}},|u-\gamma w| \ll 1$, so one sees that $w_{c}^{\prime}$ is essentially determined by $u_{c}$. Indeed, let $T_{0}$ be the time at which $U(\bar{c}(\varepsilon), \xi)$ enters $B$, and let $T_{1}$ be the exit time:

$$
\begin{aligned}
& \left(e^{-(\varepsilon \gamma / c) \xi} w_{c}\right)^{\prime}=-(\varepsilon / c) e^{-(e \gamma / c)}\left(u_{c}-(u-\gamma w) / c\right), \\
& e^{-(\varepsilon \gamma / c)} w_{c}\left(T_{1}\right)=e^{-(\varepsilon \gamma / c) T_{0}} w_{c}\left(T_{0}\right)-(\varepsilon / c) \int_{T_{0}}^{T_{1}} e^{-(\varepsilon \gamma / c) \xi}\left(u_{c}-\frac{u-\gamma w}{c}\right) d \xi .
\end{aligned}
$$

Since $|u-\gamma w| \ll 1, u_{c} \ll 0$ and $T_{1}-T_{0}$ is $O(1 / \varepsilon)$ (the time spent on the slow manifold), $w_{c}\left(T_{1}\right) \rightarrow-\infty$ as $\varepsilon \rightarrow 0$ (recall $c<0$ ).

The time taken by $U(\bar{c}(\varepsilon), \xi)$ between leaving $B$ and crossing $\{u=a\}$ is bounded independently of $\varepsilon$. Hence at $T=T(\varepsilon)$, if $\varepsilon$ is small enough, $u_{c}<0$ and $w_{c}<0$.

Also at $u=a, u_{\xi}<0$ and $w_{\xi}=-(\varepsilon / c)(u-\gamma w)$ with $\gamma \ll 1, w_{\xi}>0$. From this, one sees that (7.14) is negative. This implies $m>0$.

Let $K_{\varepsilon}=n N$. I must check that $n>0$. For this we need that the second component of $\eta$ is positive. As $\xi \rightarrow+\infty, \eta(0, \xi)$ is asymptotic to $Y^{-}$, whose second component is $\left(c-\beta^{-}\right)^{-1} \cdot \beta^{-}$is close to $\left(c-\left(c^{2}-4 f^{\prime}(0)\right)^{1 / 2}\right) / 2$, since $f^{\prime}(0)<0$ and $\left(c-\beta^{-}\right)^{-1}$ is positive. This component must stay positive as the solution moves up the left slow manifold. By continuity of $W^{\text {cs }}(\varepsilon)$ in $\varepsilon$, some scalar multiple of $\eta(0, \xi)$ will stay close to the normal of $L^{\mathrm{s}}$. By the same argument that shows $K_{\varepsilon} \rightarrow K_{0}$, the second component of this vector can never be zero. Therefore the second component of $\eta$ stays positive on $(T,+\infty)$. It follows that $n>0$.

I now have proved that

$$
Q_{\varepsilon}=m\left(\frac{\partial U}{\partial c}+\frac{\partial U}{\partial \xi} \tau^{\prime}, 1\right) \quad \text { and } K_{\varepsilon}=n N
$$

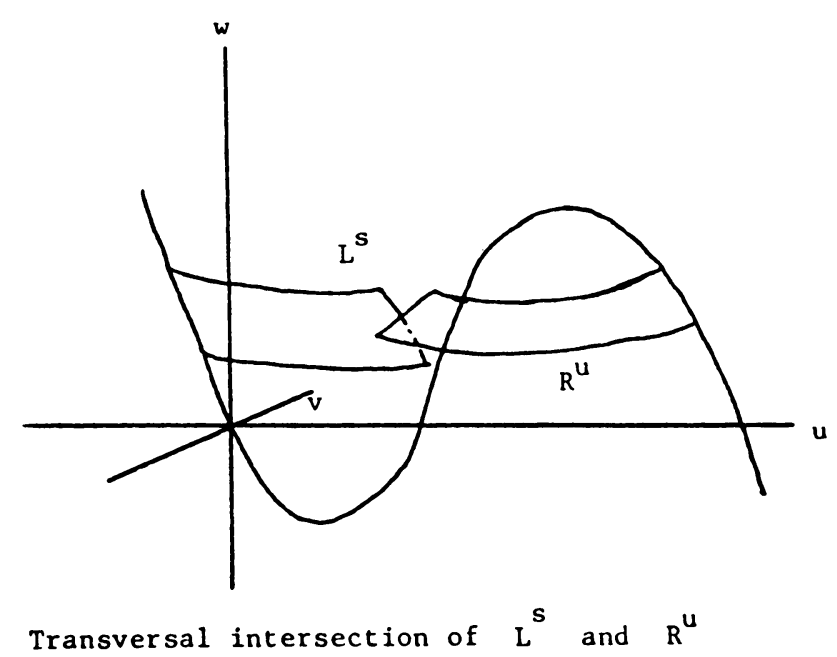

FigURE 9 
with both $m, n>0$. Furthermore,

$$
\begin{aligned}
Q_{\varepsilon} \cdot K_{\varepsilon} & =m n\left(\frac{\partial U}{\partial c}, 1\right) \cdot N+m n\left(\frac{\partial U}{\partial \xi} \tau^{\prime}, 0\right) \cdot N \\
& =m n\left(\frac{\partial U}{\partial c} \cdot \eta+k\right)+m n \tau^{\prime}\left(\frac{\partial U}{\partial \xi} \cdot \eta\right),
\end{aligned}
$$

but $\partial U / \partial \xi \cdot \eta=0$, since 0 is an eigenvalue and $\partial U / \partial \xi$ is an eigenfunction. Therefore,

$$
Q_{\varepsilon} \cdot K_{\varepsilon}=m n(P(T) \cdot N(T))
$$

Since $m, n>0$,

$$
\operatorname{sgn}\left(Q_{\varepsilon} \cdot K_{\varepsilon}\right)=\operatorname{sgn}(P(T) \cdot N(T)) .
$$

Since $Q_{\varepsilon} \rightarrow Q_{0}$ and $K_{\varepsilon} \rightarrow K_{0}$, the lemma follows, as desired.

It now remains to prove Lemma 7.1. This will depend on an argument given by Langer, which, in this case, applies exactly, since $\varepsilon=0$, and the value of $\gamma$ is therefore irrelevant.

Proof of Lemma 7.1. To compute $Q_{0} \cdot K_{0}$ we can project onto $\mathbf{R}^{3}$ as the fourth component of $Q_{0}$ is zero. $Q_{0}$ is tangent to $R^{u} \cap\{c=\bar{c}(0)\} \cap\{u=a\}$ and has a negative $w$ component. $R^{u}$ can be expressed as the graph of a function $v=h(u, w)$ in $\mathbf{R}^{3}$ near $\omega(0) . Q_{0}$ is therefore tangent to the curve $(a, h(a, w), w)$, and so a multiple of $\left(0, h_{w}, 1\right)$. It is a positive multiple of $\left(0,-h_{w},-1\right)$.

Now $K_{0}$, projected onto $c=\bar{c}(0)$, is normal to $W^{\mathrm{s}}(\varepsilon) ; c=\bar{c}(\varepsilon)$ is given by $v=g(u, w)$. A normal with positive $v$ component is therefore $\left(-g_{u}, 1,-g_{w}\right)$. It follows that $Q_{0} \cdot K_{0}$ is a positive multiple of $g_{u^{*}}-h_{n}$. Langer proves the inequalities

$$
h_{n}>0 \text { and } g_{n}<0,
$$

from which the lemma follows.

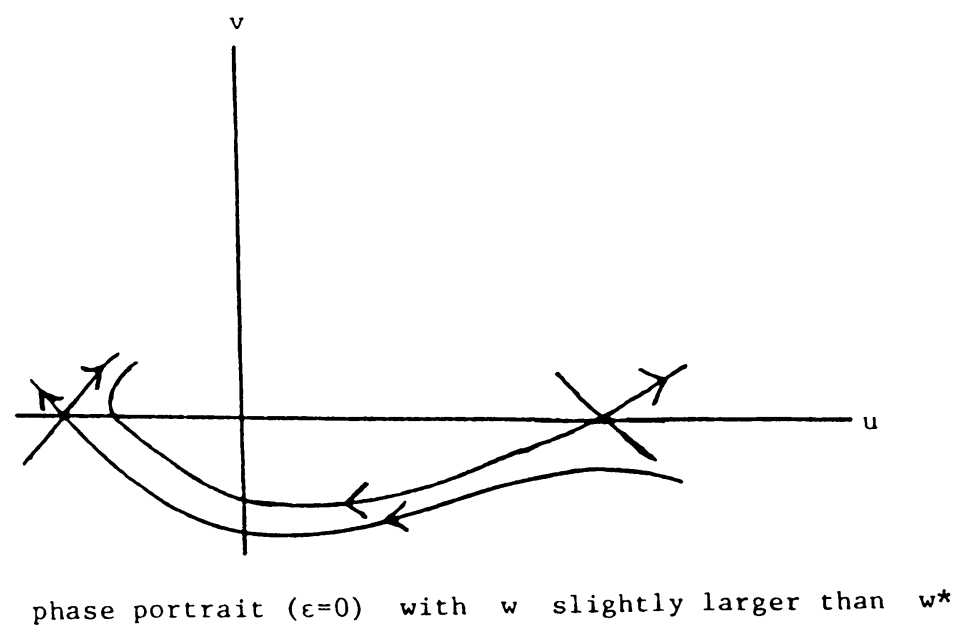

Figure 10 
REMARK. Inequalities (7.16) have a very pretty and important geometrical interpretation. They quantify how the unstable manifold from the right slow manifold meets the stable manifold of the left one; see Figure 9. The direction is determined by the way the connection breaks as $w$ changes. The phase portrait for $w$ slightly larger than $w^{*}$ is given in Figure 10. This is then easily seen to be the content of (7.16).

\section{REFERENCES}

1. M. Bramson, Kolmogorov nonlinear diffusion equations, Mem. Amer. Math. Soc. (to appear).

2. G. Carpenter, A geometric approach to singular perturbation problems with applications to nerie impulse equations. J. Differential Equations 23 (1977), 335-367.

3. C. Conley, On travelling wave solutions of nonlinear diffusion equations, Dynamical Systems Theory and Applications (J. Moser, ed.), Lecture Notes in Physics, Vol. 38, Springer-Verlag, Berlin. 1975.

4. C. Conley and R. Gardner, An application of the generalized Morse index to travelling wave solutions of a competitive reaction-diffusion model, Indiana Univ. Math. J. 33 (1984), 319-343.

5. S. Dunbar, Travelling waves of diffusive Volterra-Lother interaction equations, $\mathrm{Ph}$.D. Thesis, Univ. of Minnesota, 1981.

6. J. W. Evans, Nerve axon equations. I: Linear approximations, Indiana Univ. Math. J. 21 (1972). 877-955.

7. __ Nerve axon equations. II: Stability at rest, Indiana Univ. Math. J. 22 (1972), 75-90.

8. Nerve axon equations III: Stability of the nerve impulse, Indiana Univ. Math. J. 22 (1972). $577-594$

9. Nerve axon equations. IV: The stable and the unstable impulse, Indiana Univ. Math. J. 24 (1975), 1169-1190.

10. N. Fenichel, Persistence and smoothness of invariant manifolds for flows, Indiana Univ. Math. J. 21 (1971), 193-226.

11. J. Feroe, Temporal stability of solitary impulse solutions of a nerve equation, Biophys. J. 21 (1978), 103-110.

12. P. Fife, Mathematical aspects of reacting and diffusing systems, Lecture Notes in Biomath., Vol. 28. Springer-Verlag, Berlin, 1979.

13. P. Fife and J. B. McLeod, The approach of solutions of nonlinear diffusion equations to travelling front solutions, Arch. Rational Mech. Anal. 65 (1977), 335-361.

14. R. FitzHugh, Impulses and physiological states in theoretical models of nerve membranes, Biophys. J. 1 (1961), 445-466.

15. R. Gardner, Existence and stability of travelling wave solutions of competition models: a degree theoretic approach, J. Differential Equations 44 (1982), 343-364.

16. R. Gardner and J. Smoller, The existence of periodic travelling waves for singularly perturbed predator-prey equations via the Conley index, J. Differential Equations 47 (1983), 133-161.

17. S. P. Hastings, On the existence of homoclinic and periodic orbits for the FitzHugh-Nagumo equations, Quart. J. Math. Oxford Ser. (2) 27 (1976), 123-134.

18. D. Henry, The geometric theory of semilinear parabolic equations, Lecture Notes in Math., Vol. 840. Springer-Verlag, Berlin, 1981.

19. G. Klaasen and W. Troy, The stability of travelling wave front solutions of a reaction-diffusion system. SIAM J. Appl. Math. 41 (1981), 145-167.

20. R. Langer, Existence of homoclinic travelling wave solutions to the FitzHugh-Nagumo equations. Ph.D. Thesis, Northeastern Univ., 1980.

21. J. Nagumo, S. Arimoto and S. Yoshizawa, An active pulse transmission line simulating nerve axons, Proc. IRL SO (1960), 2061-2070.

22. J. Rauch and J. Smoller, Qualitative theory of the FitzHugh-Nagumo equations, Adv. in Math. 27 (1978), 12-44.

23. D. Terman, Threshold phenomena in nonlinear diffusion equations, Ph.D. Thesis, Univ. of Minnesota, 1980.

Department of Mathematics, University of Arizona, Tucson, Arizona 85721 